\title{
TORSION IN THE 0-CYCLE GROUP WITH MODULUS
}

\author{
AMALENDU KRISHNA
}

\begin{abstract}
We show, for a smooth projective variety $X$ over an algebraically closed field $k$ with an effective Cartier divisor $D$, that the torsion subgroup $\mathrm{CH}_{0}(X \mid D)\{l\}$ can be described in terms of a relative étale cohomology for any prime $l \neq p=\operatorname{char}(k)$. This extends a classical result of Bloch, on the torsion in the ordinary Chow group, to the modulus setting. We prove the Roitman torsion theorem (including $p$-torsion) for $\mathrm{CH}_{0}(X \mid D)$ when $D$ is reduced. We deduce applications to the problem of invariance of the prime-to- $p$ torsion in $\mathrm{CH}_{0}(X \mid D)$ under an infinitesimal extension of $D$.
\end{abstract}

\section{Contents}

1. Introduction

2. Review of 0-cycle groups and Albanese varieties

3. Torsion in the Chow group of a singular surface

4. Roitman's torsion for separably weakly normal surfaces

5. Bloch's torsion theorem for 0-cycles with modulus

6. Roitman's torsion theorem for 0-cycles with modulus

References

\section{INTRODUCTION}

One of the fundamental problems in the study of the theory of motives, as envisioned by Grothendieck, is to construct a universal cohomology theory of varieties and describe it in terms of algebraic cycles. When $X$ is a smooth quasi-projective scheme over a base field $k$, such a motivic cohomology theory of $X$ is known to exist (see, for example, [60], 39]). Moreover, Bloch [12] showed that this cohomology theory has an explicit description in terms of groups of algebraic cycles, called the higher Chow groups. These groups have all the properties that one expects, including Chern classes and a Chern character isomorphism from the higher $K$-groups of Quillen.

However, the situation becomes much complicated when we go beyond the world of smooth varieties. There is no theory of motivic cohomology of singular schemes known till date which could be a universal cohomology theory, which could be described in terms of algebraic cycles and, which could be rationally isomorphic to the algebraic $K$-theory.

With the aim of understanding the algebraic $K$-theory of the ring $k[t] /\left(t^{n}\right)$ in terms of algebraic cycles, Bloch and Esnault introduced the idea of algebraic cycles with modulus, called additive Chow groups. This idea was substantially expanded in the further works of Park [51, Rülling [55] and Krishna-Levine [32.

In recent works of Binda-Saito [8] and Kerz-Saito [29], a theory of higher Chow groups with modulus was introduced, which generalizes the construction of the additive higher

2010 Mathematics Subject Classification. Primary 14C25; Secondary 13F35, 14F30, $19 \mathrm{E} 15$.

Key words and phrases. cycles with modulus, cycles on singular schemes, algebraic $K$-theory, étale cohomology. 
Chow groups. These groups, denoted $\mathrm{CH}^{*}(X \mid D, *)$, are designed to study the arithmetic and geometric properties of a smooth variety $X$ with fixed conditions along an effective (possibly non-reduced) Cartier divisor $D$ on it (see [29]), and are supposed to give a cycle-theoretic description of the relative $K$-groups $K_{*}(X, D)$, defined as the homotopy groups of the homotopy fiber of the restriction map $K(X) \rightarrow K(D)$ (see [28]).

In order to provide evidence that the Chow groups with modulus are the right motivic cohomology groups to compute the relative $K$-theory of a smooth scheme with respect to an effective divisor, one would like to know if these groups share enough of the known structural properties of the Chow groups without modulus, and, if these groups could be related to other cohomological invariants of a pair $(X, D)$. In particular, one would like to know if the torsion part of the Chow group of 0 -cycles with modulus could be described in terms of a relative étale cohomology or, in terms of an Albanese variety with modulus.

1.1. The main results. For the Chow group of 0 -cycles without modulus on a smooth projective scheme, Bloch [10] showed that its prime-to- $p$ torsion is completely described in terms of an étale cohomology. Roitman [54] and Milne [48] showed that the torsion in the Chow group is completely described in terms of the Albanese of the underlying scheme. When $D$ is an effective Cartier divisor on a smooth projective scheme $X$, the Albanese variety with modulus $A^{d}(X \mid D)$ with appropriate universal property and the Abel-Jacobi map $\rho_{X \mid D}: \mathrm{CH}_{0}(X \mid D)_{\operatorname{deg} 0} \rightarrow A^{d}(X \mid D)$ were constructed in [7].

The goal of this work is to extend the torsion results of Bloch and Roitman-Milne to 0 -cycles with modulus. Our main results thus broadly look as follows. Their precise statements and underlying hypotheses and notations will be explained at appropriate places in the text.

Theorem 1.1. (Bloch's torsion theorem) Let $k$ be an algebraically closed field of exponential characteristic $p$. Let $X$ be a smooth projective scheme of dimension $d \geq 1$ over $k$ and let $D \subset X$ be an effective Cartier divisor. Then, for any prime $l \neq p$, there is an isomorphism

$$
\lambda_{X \mid D}: \mathrm{CH}_{0}(X \mid D)\{l\} \stackrel{\simeq}{\rightarrow} H_{e ́ t}^{2 d-1}\left(X \mid D, \mathbb{Q}_{l} / \mathbb{Z}_{l}(d)\right) .
$$

Theorem 1.2. (Roitman's torsion theorem) Let $k$ be an algebraically closed field of exponential characteristic $p$. Let $X$ be a smooth projective scheme of dimension $d \geq 1$ over $k$ and let $D \subset X$ be an effective Cartier divisor. Assume that $D$ is reduced. Then, the Albanese variety with modulus $A^{d}(X \mid D)$ is a semi-abelian variety and the Abel-Jacobi map $\rho_{X \mid D}: \mathrm{CH}_{0}(X \mid D)_{\operatorname{deg} 0} \rightarrow A^{d}(X \mid D)$ is an isomorphism on the torsion subgroups (including $p$-torsion).

Note that the isomorphism of Theorem 1.1 is very subtle because one does not have any obvious map in either direction. The construction of such a map is an essential part of the problem. In the case of Chow group without modulus, the construction of $\lambda_{X}$ by Bloch makes essential use of the Bloch-Ogus theory and, more importantly, it uses the Weil-conjecture. The prime-to- $p$ part of Theorem 1.2 was earlier proven in [7]. The new contribution here is the description of the more challenging $p$-part.

As part of the proof of Theorem 1.2, we prove the Roitman torsion theorem (including $p$-torsion) for singular separably weakly normal surfaces (see Definition 4.5). This provides the first evidence that the Roitman torsion theorem may be true for non-normal varieties in positive characteristic.

Theorem 1.3. Let $X$ be a reduced projective separably weakly normal surface over an algebraically closed field $k$ of exponential characteristic $p$. Then, the Albanese variety $A^{2}(X)$ is a semi-abelian variety and the Abel-Jacobi map $\rho_{X}: \mathrm{CH}_{0}(X)_{\operatorname{deg} 0} \rightarrow A^{2}(X)$ is an isomorphism on the torsion subgroups (including p-torsion). 
Note that Theorem 1.2 makes no assumption on $\operatorname{dim}(X)$ but this is not the case for Theorem 1.3. The reason for this is that our proof of Theorem 1.2 uses a weaker version of the Lefschetz type theorem for separably weakly normal varieties. We do not know if a general hyperplane section of an arbitrary separably weakly normal variety is separably weakly normal. This is known to be false for weakly normal varieties in positive characteristic (see [16]). We shall investigate this question in a future project.

1.2. Applications. As an application of Theorem 1.1, we obtain the following result about the prime-to- $p$ torsion in the Chow group with modulus.

Corollary 1.4. Let $k$ be an algebraically closed field of exponential characteristic p. Let $X$ be a smooth projective scheme of dimension $d \geq 1$ over $k$ and let $D \subset X$ be an effective Cartier divisor. Then, the restriction map ${ }_{n} \mathrm{CH}_{0}(X \mid D) \rightarrow{ }_{n} \mathrm{CH}_{0}\left(X \mid D_{\text {red }}\right)$ is an isomorphism for every integer $n$ prime to $p$.

In [49, Theorem 1.3], an isomorphism of similar indication has been very recently shown after inverting $\operatorname{char}(k)$. But it has no implication on Corollary 1.4. Another application of Theorem 1.1 is the following extension of the results of Bloch [11, Chap. 5] to the relative $K$-theory.

Corollary 1.5. Let $X$ be a smooth projective surface over an algebraically closed field $k$ of exponential characteristic $p$ and let $l \neq p$ be a prime. Let $D \subset X$ be an effective Cartier divisor. Then, the following hold.

(1) $H^{1}\left(X, \mathcal{K}_{2,(X, D)}\right) \otimes_{\mathbb{Z}} \mathbb{Q}_{l} / \mathbb{Z}_{l}=0$.

(2) $H^{2}\left(X, \mathcal{K}_{2,(X, D)}\right)\{l\} \simeq H_{\text {ét }}^{3}\left(X \mid D, \mathbb{Q}_{l} / \mathbb{Z}_{l}(2)\right)$.

Analogous results are also obtained for surfaces with arbitrary singularities (see Theorems 3.4 and 3.6).

1.3. Outline of proofs. Our proofs of the above results are based on the decomposition theorem of [7] which relates the Chow group of 0 -cycles with modulus on a smooth variety to the Levine-Weibel Chow group of 0 -cycles on a singular variety. Using this decomposition theorem, our main task becomes extending the torsion results of Bloch and Roitman-Milne to singular varieties. We prove these results for the Chow group of 0 -cycles on singular surfaces in the first part $(\S 3$ and $\S 4)$ of this text. These results are of independent interest in the study of 0-cycles on singular varieties.

In $\S[$ and $\S[$, we prove our results about 0 -cycles with modulus by using the analogous results for singular surfaces and an induction on dimension. This induction is based on some variants of the Lefschetz hyperplane section theorem for the Albanese variety and the weak Lefschetz theorem for the étale cohomology with modulus. Some applications are deduced in $\S 5$.

1.4. Notations. Throughout this text (except in $\S$ 4.1), $k$ will denote an algebraically closed field of exponential characteristic $p \geq 1$. We shall denote the category of separated schemes of finite type over $k$ by $\mathbf{S c h}_{k}$. The subcategory of $\mathbf{S c h}_{k}$ consisting of smooth schemes over $k$ will be denoted by $\mathbf{S m}_{k}$. An undecorated product $X \times Y$ in $\mathbf{S c h}_{k}$ will mean that it is taken over the base scheme $\operatorname{Spec}(k)$. An undecorated cohomology group will be assumed to be with respect to the Zariski site. We shall let $w: \mathbf{S c h}_{k} / e ́ t \rightarrow$ $\mathbf{S c h}_{k} /$ zar denote the canonical morphism between the étale and the Zariski sites of $\mathbf{S c h}_{k}$. For $X \in \mathbf{S c h}_{k}$, the notation $X^{N}$ will usually mean the normalization of $X_{\text {red }}$ unless we use a different notation in a specific context. For any abelian group $A$ and an integer $n \geq 1$, the subgroup of $n$-torsion elements in $A$ will be denoted by ${ }_{n} A$ and, for any prime $l$, the $l$-primary torsion subgroup will be denoted by $A\{l\}$. 


\section{Review of 0-Cycle groups And Albanese varieties}

To prove the main results of $\S 1$, our strategy is to use the decomposition theorem for 0 -cycles from [7]. This theorem asserts that the Chow group of 0-cycles with modulus on a smooth scheme is a direct summand of the Chow group of 0-cycles (in the sense of [41]) on a singular scheme. We therefore need to prove analogues of Theorems 1.1 and 1.2 for singular schemes. In the first few sections of this paper, our goal is to prove such results for arbitrary singular surfaces. These results are of independent interest and answer some questions in the study of 0-cycles on singular schemes. The higher dimensional cases of Theorems 1.1 and 1.2 will be deduced from the case of surfaces.

In this section, we review the definitions and some basic facts about the Chow group of 0 -cycles on singular schemes, the Chow group with modulus and their universal quotients, called the Albanese varieties.

2.1. 0-cycles on singular schemes. We recall the definition of the cohomological Chow group of 0 -cycles for singular schemes from [7] and [41]. Let $X$ be a reduced quasi-projective scheme of dimension $d \geq 1$ over $k$. Let $X_{\text {sing }}$ and $X_{\text {reg }}$ denote the loci of the singular and the regular points of $X$. Given a nowhere dense closed subscheme $Y \subset X$ such that $X_{\text {sing }} \subseteq Y$ and no component of $X$ is contained in $Y$, we let $\mathcal{Z}_{0}(X, Y)$ denote the free abelian group on the closed points of $X \backslash Y$. We write $\mathcal{Z}_{0}\left(X, X_{\text {sing }}\right)$ in short as $\mathcal{Z}_{0}(X)$.

Definition 2.1. Let $C$ be a pure dimension one reduced scheme in $\mathbf{S c h}_{k}$. We shall say that a pair $(C, Z)$ is a good curve relative to $X$ if there exists a finite morphism $\nu: C \rightarrow X$ and a closed proper subscheme $Z \subsetneq C$ such that the following hold.

(1) No component of $C$ is contained in $Z$.

(2) $\nu^{-1}\left(X_{\text {sing }}\right) \cup C_{\text {sing }} \subseteq Z$.

(3) $\nu$ is local complete intersection at every point $x \in C$ such that $\nu(x) \in X_{\text {sing. }}$.

Let $(C, Z)$ be a good curve relative to $X$ and let $\left\{\eta_{1}, \cdots, \eta_{r}\right\}$ be the set of generic points of $C$. Let $\mathcal{O}_{C, Z}$ denote the semilocal ring of $C$ at $S=Z \cup\left\{\eta_{1}, \cdots, \eta_{r}\right\}$. Let $k(C)$ denote the ring of total quotients of $C$ and write $\mathcal{O}_{C, Z}^{\times}$for the group of units in $\mathcal{O}_{C, Z}$. Notice that $\mathcal{O}_{C, Z}$ coincides with $k(C)$ if $|Z|=\emptyset$. As $C$ is Cohen-Macaulay, $\mathcal{O}_{C, Z}^{\times}$is the subgroup of $k(C)^{\times}$consisting of those $f$ which are regular and invertible in the local rings $\mathcal{O}_{C, x}$ for every $x \in Z$.

Given any $f \in \mathcal{O}_{C, Z}^{\times} \hookrightarrow k(C)^{\times}$, we denote by $\operatorname{div}_{C}(f)$ (or $\operatorname{div}(f)$ in short) the divisor of zeros and poles of $f$ on $C$, which is defined as follows. If $C_{1}, \ldots, C_{r}$ are the irreducible components of $C$, and $f_{i}$ is the factor of $f$ in $k\left(C_{i}\right)$, we set $\operatorname{div}(f)$ to be the 0 -cycle $\sum_{i=1}^{r} \operatorname{div}\left(f_{i}\right)$, where $\operatorname{div}\left(f_{i}\right)$ is the usual divisor of a rational function on an integral curve in the sense of [20]. As $f$ is an invertible regular function on $C$ along $Z, \operatorname{div}(f) \in$ $\mathcal{Z}_{0}(C, Z)$.

By definition, given any good curve $(C, Z)$ relative to $X$, we have a push-forward map $\mathcal{Z}_{0}(C, Z) \stackrel{\nu_{*}}{\rightarrow} \mathcal{Z}_{0}(X)$. We shall write $\mathcal{R}_{0}(C, Z, X)$ for the subgroup of $\mathcal{Z}_{0}(X)$ generated by the set $\left\{\nu_{*}(\operatorname{div}(f)) \mid f \in \mathcal{O}_{C, Z}^{\times}\right\}$. Let $\mathcal{R}_{0}(X)$ denote the subgroup of $\mathcal{Z}_{0}(X)$ generated by the image of the map $\mathcal{Z}_{0}(C, Z, X) \rightarrow \mathcal{Z}_{0}(X)$, where $(C, Z)$ runs through all good curves relative to $X$. We let $\mathrm{CH}_{0}(X)=\frac{\mathcal{Z}_{0}(X)}{\mathcal{R}_{0}(X)}$.

If we let $\mathcal{R}_{0}^{L W}(X)$ denote the subgroup of $\mathcal{Z}_{0}(X)$ generated by the divisors of rational functions on good curves as above, where we further assume that the map $\nu: C \rightarrow X$ is a closed immersion, then the resulting quotient group $\mathcal{Z}_{0}(X) / \mathcal{R}_{0}^{L W}(X)$ is denoted by $\mathrm{CH}_{0}^{L W}(X)$. Such curves on $X$ are called the Cartier curves. There is a canonical surjection $\mathrm{CH}_{0}^{L W}(X) \rightarrow \mathrm{CH}_{0}(X)$. The Chow group $\mathrm{CH}_{0}^{L W}(X)$ was discovered by Levine and Weibel [41] in an attempt to describe the Grothendieck group of a singular scheme 
in terms of algebraic cycles. The modified version $\mathrm{CH}_{0}(X)$ was introduced in [7]. As an application of our main results, we shall be able to identify the two Chow groups for certain singular schemes (see Theorem 6.6).

2.2. The Chow group of the double. Let $X$ be a smooth quasi-projective scheme of dimension $d$ over $k$ and let $D \subset X$ be an effective Cartier divisor. Recall from [7, $\S 2.1]$ that the double of $X$ along $D$ is a quasi-projective scheme $S(X, D)=X \amalg_{D} X$ so that

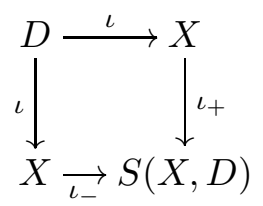

is a co-Cartesian square in $\mathbf{S c h}_{k}$. In particular, the identity map of $X$ induces a finite map $\Delta: S(X, D) \rightarrow X$ such that $\Delta \circ \iota_{ \pm}=\operatorname{Id}_{X}$ and $\pi=\iota_{+} \amalg \iota_{-}: X \amalg X \rightarrow S(X, D)$ is the normalization map. We let $X_{ \pm}=\iota_{ \pm}(X) \subset S(X, D)$ denote the two irreducible components of $S(X, D)$. We shall often write $S(X, D)$ as $S_{X}$ when the divisor $D$ is understood. $S_{X}$ is a reduced quasi-projective scheme whose singular locus is $D_{\text {red }} \subset S_{X}$. It is projective whenever $X$ is so. The following easy algebraic lemma shows that (2.1) is also a Cartesian square.

Lemma 2.2. Let

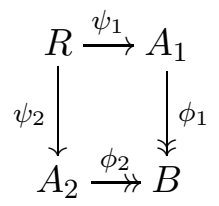

be a Cartesian square of commutative Noetherian rings such that $\operatorname{Ker}\left(\phi_{i}\right)=\left(a_{i}\right)$ for $i=1,2$. Then $A_{1} \otimes_{R} A_{2} \stackrel{\simeq}{\rightarrow} B$.

Proof. If we let $\alpha_{1}=\left(0, a_{2}\right)$ and $\alpha_{2}=\left(a_{1}, 0\right)$ in $R \subset A_{1} \times A_{2}$, then it is easy to check that for $i=1,2$, the map $\psi_{i}$ is surjective and $\operatorname{Ker}\left(\psi_{i}\right)=\left(\alpha_{i}\right)$. This implies that $A_{1} \otimes_{R} A_{2} \simeq R /\left(\alpha_{1}\right) \otimes_{R} A_{2} \simeq A_{2} /\left(a_{2}\right)=B$.

We shall later consider a more general situation than (2.1) where we allow the two components of the double to be distinct. This general case is a non-affine version of (2.2) and is used in the proof of Theorem 1.2 .

2.3. The Albanese varieties for singular schemes. Let $X$ be a reduced projective scheme over $k$ of dimension $d$. Let $\mathrm{CH}_{0}^{L W}(X)_{\operatorname{deg} 0}$ denote the kernel of the degree map $\operatorname{deg}: \mathrm{CH}_{0}^{L W}(X) \rightarrow H^{0}(X, \mathbb{Z})$. An Albanese variety $A^{d}(X)$ of $X$ was constructed in [18]. This variety is a connected commutative algebraic group over $k$ with an Abel-Jacobi map $\rho_{X}: \mathrm{CH}_{0}^{L W}(X)_{\operatorname{deg} 0} \rightarrow A^{d}(X)$. Moreover, $A^{d}(X)$ is the universal object in the category of commutative algebraic groups $G$ over $k$ with a rational map $f: X \rightarrow G$ which induces a group homomorphism $f_{*}: \mathrm{CH}_{0}^{L W}(X)_{\operatorname{deg} 0} \rightarrow G$. Such a group homomorphism is called a regular homomorphism. For this reason, $A^{d}(X)$ is also called the universal regular quotient of $\mathrm{CH}_{0}^{L W}(X)_{\operatorname{deg} 0}$. When $X$ is smooth, $A^{d}(X)$ coincides with the classical Albanese variety $\operatorname{Alb}(X)$. One says that $\mathrm{CH}_{0}^{L W}(X)_{\operatorname{deg} 0}$ is finite-dimensional, if $\rho_{X}$ is an isomorphism. 
2.4. The universal semi-abelian quotient of $\mathrm{CH}_{0}^{L W}(X)_{\operatorname{deg} 0}$. In positive characteristic, apart from the theorem about its existence, not much is known about $A^{d}(X)$ and almost everything that one would like to know about it is an open question. However, the universal semi-abelian quotient of the algebraic group $A^{d}(X)$ can be described more explicitly and this is all one needs to know in order to understand the prime-to- $p$ torsion in $A^{d}(X)$. The following description of this quotient is recalled from [42, $\left.\S 2\right]$.

Let $\pi: X^{N} \rightarrow X$ denote the normalization map. Let $\mathrm{Cl}\left(X^{N}\right)$ and $\operatorname{Pic}_{W}\left(X^{N}\right)$ denote the class group and the Weil Picard group of $X^{N}$. Recall from 61 that $\operatorname{Pic}_{W}\left(X^{N}\right)$ is the subgroup of $\mathrm{Cl}\left(X^{N}\right)$ consisting of Weil divisors which are algebraically equivalent to zero in the sense of [20, Chap. 19]. Let $\operatorname{Div}(X)$ denote the free abelian group of Weil divisors on $X$. Let $\Lambda_{1}(X)$ denote the subgroup of $\operatorname{Div}\left(X^{N}\right)$ generated by the Weil divisors which are supported on $\pi^{-1}\left(X_{\text {sing }}\right)$. This gives us a map $\iota_{X}: \Lambda_{1}(X) \rightarrow \frac{\mathrm{Cl}\left(X^{N}\right)}{\operatorname{Pic}_{W}\left(X^{N}\right)}$.

Let $\Lambda(X)$ denote the kernel of the canonical map

$$
\Lambda_{1}(X) \stackrel{\left(\iota_{X}, \pi_{*}\right)}{\longrightarrow} \frac{\mathrm{Cl}\left(X^{N}\right)}{\operatorname{Pic}_{W}\left(X^{N}\right)} \oplus \operatorname{Div}(X) .
$$

It follows from [42, $\S 4$ ] that the universal semi-abelian quotient of $A^{d}(X)$ is the Cartier dual of the 1-motive $\left[\Lambda(X) \rightarrow \operatorname{Pic}_{W}\left(X^{N}\right)\right]$ and is denoted by $J^{d}(X)$. The composite homomorphism $\rho_{X}^{\text {semi }}: \mathrm{CH}_{0}^{L W}(X)_{\operatorname{deg} 0} \rightarrow A^{d}(X) \rightarrow J^{d}(X)$ is universal among regular homomorphisms from $\mathrm{CH}_{0}^{L W}(X)_{\operatorname{deg} 0}$ to semi-abelian varieties. $J^{d}(X)$ is called the semiabelian Albanese variety of $X$. By [7, Proposition 9.7], there is a factorization of $\rho_{X}^{\text {semi }}$ :

$$
\mathrm{CH}_{0}^{L W}(X)_{\operatorname{deg} 0} \rightarrow \mathrm{CH}_{0}(X)_{\operatorname{deg} 0} \stackrel{\tilde{\rho}_{X}^{\text {semi }}}{\longrightarrow} J^{d}(X)
$$

and $J^{d}(X)$ is in fact a universal regular semi-abelian variety quotient of $\mathrm{CH}_{0}(X) \operatorname{deg} 0$.

There is a short exact sequence of commutative algebraic groups

$$
0 \rightarrow A_{\text {unip }}^{d}(X) \rightarrow A^{d}(X) \rightarrow J^{d}(X) \rightarrow 0,
$$

where $A_{\text {unip }}^{d}(X)$ is the unipotent radical of $A^{d}(X)$. Since $k$ is algebraically closed, $A_{\text {unip }}^{d}(X)$ is uniquely $n$-divisible for any integer $n \in k^{\times}$. In particular,

$$
{ }_{n} A^{d}(X) \stackrel{\simeq}{\rightarrow} J^{d}(X) .
$$

2.5. A Lefschetz hyperplane theorem. Recall that for a smooth projective scheme $X \hookrightarrow \mathbb{P}_{k}^{N}$ of dimension $d \geq 3$, a general hypersurface section $Y \subset X$ has the property that the canonical map $\operatorname{Alb}(Y) \rightarrow \operatorname{Alb}(X)$ is an isomorphism. We now wish to prove a similar result for the semi-abelian Albanese variety $J^{d}\left(S_{X}\right)$ of the double.

Let $X$ be a connected smooth projective scheme of dimension $d \geq 3$ over $k$ and let $D \subset X$ be an effective Cartier divisor. Let $\left\{E_{1}, \cdots, E_{r}\right\}$ be the set of irreducible components of $D_{\text {red }}$ so that each $E_{i}$ is integral. For each $1 \leq j \leq r$, let $P_{j} \in E_{j} \backslash\left(\cup_{j^{\prime} \neq j} E_{j^{\prime}}\right)$ be a chosen closed point, and let $S=\left\{P_{1}, \cdots, P_{r}\right\}$. We can apply [1, Theorems 1, 7] to find a closed embedding $X \hookrightarrow \mathbb{P}_{k}^{N}$ (for $N \gg 0$ ) such that for a general set of distinct hypersurfaces $H_{1}, \cdots, H_{d-2} \hookrightarrow \mathbb{P}_{k}^{N}$, we have the following.

(1) The successive intersection $X \cap H_{1} \cap \cdots \cap H_{i}(1 \leq i \leq d-2)$ is integral and smooth over $k$.

(2) No component of $X \cap H_{1} \cap \cdots \cap H_{d-2}$ is contained in $D$.

(3) $S \subset X \cap H_{1} \cap \cdots \cap H_{d-2}$.

(4) For each $1 \leq j \leq r$, the successive intersection $E_{j} \cap H_{1} \cap \cdots \cap H_{i}(1 \leq i \leq d-2)$ is integral. 
Setting $X_{i}=X \cap H_{1} \cap \cdots \cap H_{i}$ and $D_{i}=X_{i} \cap D$, our choice of the hypersurfaces implies that $D_{i} \subset X_{i}$ is an effective Cartier divisor. We let $S_{i}=X_{i} \amalg_{D_{i}} X_{i}$ for $1 \leq i \leq d-2$. It follows from [7, Proposition 2.4] that each inclusion $\tau_{i}: S_{i} \hookrightarrow S_{i-1}$ is a local complete intersection (l.c.i.) and $S_{i}=S_{i-1} \times_{X_{i-1}} X_{i}$ for $1 \leq i \leq d-2$, where we let $S_{0}=S_{X}$. Since $S_{i} \hookrightarrow S_{i-1}$ is an l.c.i. inclusion with $\left(S_{i}\right)_{\text {sing }}=\left(D_{i}\right)_{\text {red }}=\left(S_{i} \cap D\right)_{\text {red }}=S_{i} \cap\left(S_{i-1}\right)_{\text {sing }}$, it follows that there are compatible push-forward maps $\tau_{i, *}^{L W}: \mathrm{CH}_{0}^{L W}\left(S_{i}\right) \rightarrow \mathrm{CH}_{0}^{L W}\left(S_{i-1}\right)$ (see [18, Lemma 1.8]) and $\tau_{i, *}: \mathrm{CH}_{0}\left(S_{i}\right) \rightarrow \mathrm{CH}_{0}\left(S_{i-1}\right)$ (see [7, Proposition 3.17]). With the above set-up, we have the following.

Proposition 2.3. For $1 \leq i \leq d-2$, there is an isomorphism of algebraic groups $\phi_{i}: J^{d-i}\left(S_{i}\right) \rightarrow J^{d-i+1}\left(S_{i-1}\right)$ and a commutative diagram

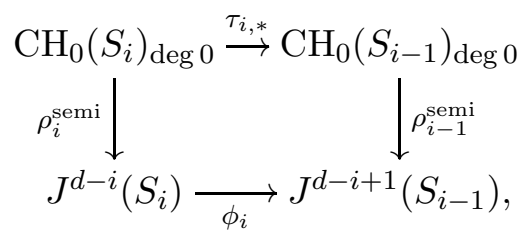

whose restriction to the l-primary torsion subgroups induces isomorphism of all arrows, for every prime $l \neq p$.

Proof. Before we begin the proof, we note that when $S_{X} \cap H \hookrightarrow S_{X}$ is a general hypersurface section in a projective embedding of $S_{X}$, then an analogue of the proposition was proven by Mallick in [42, §5]. But we can not apply his result directly because $S_{Y} \hookrightarrow S_{X}$ is not a hypersurface section. Nevertheless, we shall closely follow Mallick's construction in the proof of the proposition. Also, we shall prove a stronger assertion than in [42, Theorem 14] because of the special nature of the double.

We have seen above that the inclusion $S_{i} \hookrightarrow S_{i-1}$ is l.c.i. which preserves the singular loci, and hence there is a push-forward $\tau_{i, *}: \mathrm{CH}_{0}\left(S_{i}\right) \rightarrow \mathrm{CH}_{0}\left(S_{i-1}\right)$. We now construct the map $\phi_{i}$. We construct this map for $i=1$ as the method in the general case is completely identical. With this reduction in mind, we shall let $Y=X_{1}=X \cap H_{1}, F=$ $D_{1}=D \cap H_{1}$ so that $S_{1}=S_{Y}=S(Y, F)$.

Since $Y \hookrightarrow X$ is a general hypersurface section with $S_{Y}^{N}=Y \amalg Y$ and $S_{X}^{N}=X \amalg X$, one knows that the map $\operatorname{Pic}_{W}\left(S_{Y}^{N}\right)^{\vee}=\operatorname{Alb}(Y) \times \operatorname{Alb}(Y) \rightarrow \operatorname{Alb}(X) \times \operatorname{Alb}(X)=\operatorname{Pic}_{W}\left(S_{X}^{N}\right)^{\vee}$ is an isomorphism. We thus have to construct a pull-back map $\Lambda\left(S_{X}\right) \rightarrow \Lambda\left(S_{Y}\right)$ which is an isomorphism.

We consider the commutative square

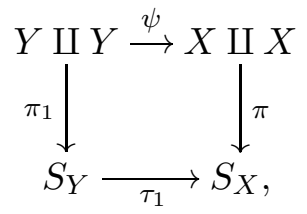

where the vertical arrows are the normalization maps and $\psi$ is the disjoint sum of the inclusion of the hypersurface section $Y=X \cap H_{1}$. We claim that this square is Cartesian. To see this, note that the vertical arrows are disjoint sums of two maps. Hence, it is enough to show that (2.9) is Cartesian when we replace $X \amalg X$ by $X_{+}$and $Y \amalg Y$ by $Y_{+}$. In this case, the vertical arrows are closed immersions and we know directly from the construction that $S_{Y} \times_{S_{X}} X_{+} \simeq\left(Y \times_{X} S_{X}\right) \times_{S_{X}} X_{+} \simeq Y_{+}$.

If we let $F_{i}:=E_{i} \cap H_{1}$ for $1 \leq j \leq r$, it follows from the conditions (2) and (3) of (2.7) that each $F_{i}$ is integral and $F_{i} \neq F_{j}$ if $E_{i} \neq E_{j}$. Now, the refined Gysin homomorphism $\psi^{!}: \Lambda_{1}\left(S_{X}\right)=\mathrm{CH}_{d-1}(D \amalg D) \rightarrow \mathrm{CH}_{d-2}(F \amalg F)=\Lambda_{1}\left(S_{Y}\right)$ (in the sense of [20]) takes $E_{i}$ to $F_{i}$ for $i=1, \cdots, r$ in each copy of $D$. This map is bijective by our choice of the hypersurface sections $Y$ and $F_{i}$ 's. 
Now, for any $1 \leq j \leq r$, we have $\psi^{!}\left(\left[E_{j}\right]\right)=\alpha_{j}\left[F_{j}\right]$ in $\Lambda_{1}\left(S_{Y}\right)=\mathrm{CH}_{d-2}(F \amalg F)$, where $\alpha_{j}$ is the intersection multiplicity of $E_{j} \times_{\mathbb{P}_{k}^{N}} H_{1}$ in $D \cdot H_{1}$. Since the composite morphism $E_{j} \hookrightarrow X \hookrightarrow \mathbb{P}_{k}^{N}$ is a closed immersion (and hence separable), this intersection multiplicity must be one so that we have $\alpha_{j}=1$ for each $j$. It follows that $\psi^{!}: \Lambda_{1}\left(S_{X}\right) \rightarrow \Lambda_{1}\left(S_{Y}\right)$ is an isomorphism of abelian groups.

We next show that $\psi^{!}$takes $\Lambda\left(S_{X}\right)$ onto $\Lambda\left(S_{Y}\right)$. This follows because for any $\Delta \in$ $\Lambda\left(S_{X}\right)$, we have $\pi_{1, *} \circ \psi^{!}(\Delta)=\tau_{1}^{!} \circ \pi_{*}(\Delta)$ by [20, Theorem 6.2] and $\pi_{*}(\Delta)=0$ in $\mathrm{CH}_{d-1}\left(\left(S_{X}\right)_{\text {sing }}\right)=Z_{d-1}\left(\left(S_{X}\right)_{\text {sing }}\right)$. This means $\psi^{!}(\Delta) \in \Lambda\left(S_{Y}\right)$. If we let $\Delta=\sum_{l} \alpha_{l}\left[F_{l}\right] \in$ $\Lambda\left(S_{Y}\right)$, then letting $\Delta^{\prime}=\sum_{l} \alpha_{l}\left[E_{l}\right] \in \Lambda_{1}\left(S_{X}\right)$, we get $\psi^{!}\left(\Delta^{\prime}\right)=\Delta$. We also have $\tau_{1}^{!} \circ \pi_{*}\left(\Delta^{\prime}\right)=\pi_{1, *} \circ \psi^{!}\left(\Delta^{\prime}\right)=\pi_{1, *}(\Delta)=0$. Since $\tau_{1}^{!}: \mathrm{CH}_{d-1}\left(\left(S_{X}\right)_{\operatorname{sing}}\right)=\mathrm{CH}_{d-1}(D) \rightarrow$ $\mathrm{CH}_{d-2}(F)=\mathrm{CH}_{d-2}\left(\left(S_{Y}\right)_{\text {sing }}\right)$ is bijective, it follows that $\pi_{*}\left(\Delta^{\prime}\right)=0$. It follows that $\psi^{!}: \Lambda\left(S_{X}\right) \rightarrow \Lambda\left(S_{Y}\right)$ is surjective and hence an isomorphism. We have thus constructed an isomorphism $\phi_{1}: J^{d-1}\left(S_{Y}\right) \stackrel{\simeq}{\rightarrow} J^{d}\left(S_{X}\right)$.

To show the commutative diagram (2.8), we can again assume $i=1$. We can also replace $\mathrm{CH}_{0}\left(S_{X}\right)$ and $\mathrm{CH}_{0}\left(S_{Y}\right)$ by $\mathcal{Z}_{0}\left(S_{X}\right)$ and $\mathcal{Z}_{0}\left(S_{Y}\right)$, respectively.

We now need to observe that $J^{d}\left(S_{X}\right)$ is a quotient of the Cartier dual $J_{\text {Serre }}^{d}\left(S_{X}\right)$ of the 1-motive $\left[\Lambda_{1}\left(S_{X}\right) \rightarrow \operatorname{Pic}_{W}(X \amalg X)\right]$ and this dual semi-abelian variety is the universal object in the category of morphisms from $\left(S_{X}\right)_{\mathrm{reg}}=(X \backslash D) \amalg(X \backslash D)$ to semi-abelian varieties (see [56]). Since $\left(S_{Y}\right)_{\mathrm{reg}}=(Y \backslash D) \amalg(Y \backslash D)=\tau_{1}^{-1}\left(\left(S_{X}\right)_{\mathrm{reg}}\right)$, it follows from this universality of $J_{\text {Serre }}^{d}\left(S_{X}\right)$ that there is a commutative diagram as in (2.8) if we replace $J^{d}\left(S_{X}\right)$ and $J^{d-1}\left(S_{Y}\right)$ by $J_{\text {Serre }}^{d}\left(S_{X}\right)$ and $J_{\text {Serre }}^{d}\left(S_{Y}\right)$, respectively. The commutative diagram

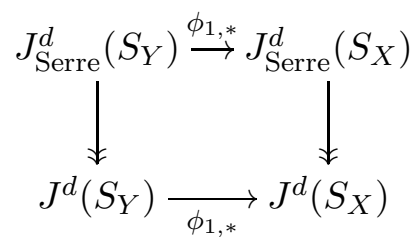

now finishes the proof of the commutativity of (2.8). The final assertion about the isomorphism between the $l$-primary torsion subgroups follows from [7, Theorem 9.8] and [42, Theorem 16].

2.6. 0-cycles and Albanese variety with modulus. Given an integral normal curve $C$ over $k$ and an effective divisor $E \subset C$, we say that a rational function $f$ on $C$ has modulus $E$ if $f \in \operatorname{Ker}\left(\mathcal{O}_{C, E}^{\times} \rightarrow \mathcal{O}_{E}^{\times}\right)$. Here, $\mathcal{O}_{C, E}$ is the semilocal ring of $C$ at the union of $E$ and the generic point of $C$. In particular, $\operatorname{Ker}\left(\mathcal{O}_{C, E}^{\times} \rightarrow \mathcal{O}_{E}^{\times}\right)$is just $k(C)^{\times}$if $|E|=\emptyset$. Let $G(C, E)$ denote the group of such rational functions.

Let $X$ be a smooth quasi-projective scheme of dimension $d \geq 1$ over $k$ and let $D \subset X$ be an effective Cartier divisor. Let $\mathcal{Z}_{0}(X \mid D)$ be the free abelian group on the set of closed points of $X \backslash D$. Let $C$ be an integral normal curve over $k$ and let $\varphi_{C}: C \rightarrow X$ be a finite morphism such that $\varphi_{C}(C) \not \subset D$. The push-forward of cycles along $\varphi_{C}$ gives a well defined group homomorphism $\tau_{C}: G\left(C, \varphi_{C}^{*}(D)\right) \rightarrow Z_{0}(X \mid D)$.

Definition 2.4. We define the Chow group $\mathrm{CH}_{0}(X \mid D)$ of 0 -cycles of $X$ with modulus $D$ to be the cokernel of the homomorphism div: $\bigoplus_{\varphi_{C}: C \rightarrow X} G\left(C, \varphi_{C}^{*}(D)\right) \rightarrow Z_{0}(X \mid D)$, where the sum is taken over the set of finite morphisms $\varphi_{C}: C \rightarrow X$ from an integral normal curve such that $\varphi_{C}(C) \not \subset D$.

It is clear from the moving lemma of Bloch that there is a canonical surjection $\mathrm{CH}_{0}(X \mid D) \rightarrow \mathrm{CH}_{0}(X)$. If $X$ is projective, we let $\mathrm{CH}_{0}(X \mid D)_{\operatorname{deg} 0}$ denote the kernel of the composite map $\mathrm{CH}_{0}(X \mid D) \rightarrow \mathrm{CH}_{0}(X) \stackrel{\text { deg }}{\longrightarrow} H^{0}(X, \mathbb{Z})$. 
If $D$ is reduced, it follows using Theorem [6.6] and the constructions of [7, $\S 11]$ that there exists an Albanese variety with modulus $A^{d}(X \mid D)$ together with a surjective AbelJacobi map $\rho_{X \mid D}: \mathrm{CH}_{0}(X \mid D)_{\operatorname{deg} 0} \rightarrow A^{d}(X \mid D)$ which is a universal regular homomorphism in a suitable sense. One says that $\mathrm{CH}_{0}(X \mid D)_{\operatorname{deg} 0}$ is finite-dimensional, if $\rho_{X \mid D}$ is an isomorphism.

\section{Torsion in the Chow group of a singular surface}

Let $X$ be a reduced projective surface over $k$. When $X$ is smooth, Suslin [57, p. 19] showed that there is a short exact sequence

$$
0 \rightarrow H^{1}\left(X, \mathcal{K}_{2}\right) \otimes_{\mathbb{Z}} \mathbb{Z} / n \rightarrow H_{e ́ t}^{3}\left(X, \mu_{n}(2)\right) \rightarrow{ }_{n} \mathrm{CH}_{0}(X) \rightarrow 0
$$

for any integer $n \in k^{\times}$. When $X$ has only isolated singularities, this exact sequence was conjectured by Pedrini and Weibel [52, (0.4)] and proven by Barbieri-Viale, Pedrini and Weibel in [3, Theorem 7.7]. Expanding on their ideas, our goal in this section is to prove such an exact sequence for surfaces with arbitrary singularities. This exact sequence will be used to prove an analogue of Theorem 1.1 for singular surfaces.

3.1. Gillet's Chern classes. Let $\mathcal{K}=\Omega B Q P$ denote the simplicial presheaf on $\mathbf{S c h}_{k}$ such that $\mathcal{K}(X)=\Omega B Q P(X)$, where $B Q P(X)$ is the Quillen's construction of the simplicial set associated to the exact category $P(X)$ of locally free sheaves on $X$. For any $i \geq 0$, let $\mathcal{K}_{i}$ denote the Zariski sheaf on $\mathbf{S c h}_{k}$ associated to the presheaf $X \mapsto$ $K_{i}(X)=\pi_{i}(\mathcal{K}(X))$.

We fix integers $d, i \geq 0$ and an integer $n \geq 1$ which is prime to the exponential characteristic $p$ of the base field $k$. Let $\mu_{n}(d)$ denote the sheaf $\mu_{n}^{\otimes d}$ on $\mathbf{S c h}_{k} /$ ét such that $\mu_{n}(X)=\operatorname{Ker}\left(\mathcal{O}^{\times}(X) \stackrel{n}{\rightarrow} \mathcal{O}^{\times}(X)\right)$. Let $\mathcal{E}_{i}$ denote the simplicial sheaf on $\mathbf{S c h}_{k} /$ zar associated, by the Dold-Kan correspondence, to the good truncation $\tau^{\leq 0} \mathbf{R} w_{*} \mu_{n}(i)[2 i]$ of the chain complex of Zariski sheaves $\mathbf{R} w_{*} \mu_{n}(i)[2 i]$. In particular, we have $\pi_{q} \mathcal{E}_{i}(X)=$ $H_{e ́ t}^{2 i-q}\left(X, \mu_{n}(i)\right)$ for $X \in \mathbf{S c h}_{k}$ and $0 \leq q \leq 2 i$.

Let $\mathcal{L}$ denote the homotopy fiber of the map of simplicial presheaves $\mathcal{K} \stackrel{n}{\rightarrow} \mathcal{K}$ so that $\pi_{q} \mathcal{L}(X)=K_{q+1}(X, \mathbb{Z} / n)$ for $q \geq-1$. Let $\mathcal{H}^{i}\left(\mu_{n}(d)\right)$ denote the Zariski sheaf $\mathbf{R}^{i} w_{*} \mu_{n}(d)$ on $\mathbf{S c h}_{k} /$ zar. It follows from Gillet's construction of the Chern classes (see [22, $\left.\S 5\right]$ ) that there is a morphism of the simplicial presheaves on $\mathbf{S c h}_{k}$ :

$$
c_{i}: \mathcal{K} \rightarrow \mathcal{E}_{i}
$$

in the homotopy category of simplicial presheaves. This map is compatible with the local to global spectral sequences for the associated Zariski sheaves so that we get a map of spectral sequences which at the $E_{2}$ level is $H^{p}\left(X, \mathcal{K}_{q}\right) \rightarrow H^{p}\left(X, \mathcal{H}^{2 i-q}\left(\mu_{n}(i)\right)\right)$ and converges to the global Chern class on $\mathcal{E}_{i}$ given by $c_{i, X}: K_{q-p}(X) \rightarrow H_{e ́ t}^{2 i-q+p}\left(X, \mu_{n}(i)\right)$. These Chern classes in fact factor through the maps $\mathcal{K} \rightarrow \Sigma \mathcal{L} \rightarrow \mathcal{E}_{i}$ (see [52, $\left.\S 2\right]$ ) so that there are Chern class maps $c_{i}: \mathcal{L} \rightarrow \Omega \mathcal{E}_{i}$ in the homotopy category of simplicial presheaves [14].

Let $\widetilde{\mathcal{K}}$ denote the simplicial presheaf on $\mathbf{S c h}_{k}$ such that $\widetilde{\mathcal{K}}(X)$ is the universal covering space of $\mathcal{K}(X)$ and let $\widetilde{\mathcal{L}}$ denote the homotopy fiber of the map $\widetilde{\mathcal{K}} \stackrel{n}{\rightarrow} \widetilde{\mathcal{K}}$. This yields $\pi_{q}(\widetilde{\mathcal{K}}(X))=K_{q}(X)$ for $q \geq 2$ and $\pi_{1}(\widetilde{\mathcal{K}}(X))=0$. Furthermore, we have

$$
\pi_{q}(\widetilde{\mathcal{L}}(X))= \begin{cases}K_{q+1}(X, \mathbb{Z} / n) & \text { if } q \geq 2 \\ K_{2}(X) / n & \text { if } q=1 \\ 0 & \text { otherwise. }\end{cases}
$$

Let $\widetilde{\mathcal{K}}^{(2)}$ denote the second layer of the Postnikov tower $\left(\cdots \rightarrow \widetilde{\mathcal{K}}^{(n)} \rightarrow \widetilde{\mathcal{K}}^{(n-1)} \rightarrow \cdots \rightarrow\right.$ $\widetilde{\mathcal{K}}^{(2)} \rightarrow \widetilde{\mathcal{K}}^{(1)} \rightarrow \widetilde{\mathcal{K}}^{(0)}=\star$ ) of $\widetilde{\mathcal{K}}$. There are compatible family of maps $f_{n}: \widetilde{\mathcal{K}} \rightarrow \widetilde{\mathcal{K}}^{(n)}$ inducing $\pi_{q} \widetilde{\mathcal{K}} \stackrel{\simeq}{\rightarrow} \pi_{q} \widetilde{\mathcal{K}}^{(n)}$ for $q \leq n$ and $\pi_{q} \widetilde{\mathcal{K}}^{(n)}=0$ for $q>n$. Let $\widetilde{\mathcal{L}}^{(2)}$ denote the 
homotopy fiber of the map $\widetilde{\mathcal{K}}^{(2)} \stackrel{n}{\rightarrow} \widetilde{\mathcal{K}}^{(2)}$ so that there is a commutative diagram of simplicial presheaves

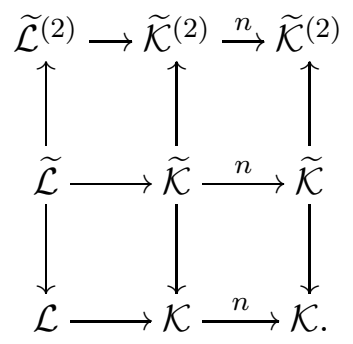

Lemma 3.1. Let $\mathcal{K}_{2}^{\bullet}$ denote the complex of presheaves $\left(\mathcal{K}_{2} \stackrel{n}{\rightarrow} \mathcal{K}_{2}\right)$ on $\mathbf{S c h}_{k} /$ zar. Then $\widetilde{\mathcal{L}}^{(2)}$ is weak equivalent to the simplicial presheaf obtained by applying the Dold-Kan correspondence to the chain complex $\mathcal{K}_{2}^{\bullet}[2]$.

Proof. Using (3.4), it suffices to show that $\widetilde{\mathcal{K}}^{(2)}$ is an Eilenberg-Mac Lane complex of the type $\left(\mathcal{K}_{2}, 2\right)$. Let $F^{2}$ denote the homotopy fiber of the Kan fibration $\widetilde{\mathcal{K}}^{(2)} \rightarrow \widetilde{\mathcal{K}}^{(1)}$ in the Postnikov tower. We have $\pi_{i} \widetilde{\mathcal{K}}^{(1)}=0$ for $i \geq 2$ and $\pi_{1} \widetilde{\mathcal{K}}^{(1)}=\pi_{1} \widetilde{\mathcal{K}}=0$ by 45 , Theorem 8.4]. The long exact homotopy sequence now implies that $\pi_{i} F^{2} \stackrel{\simeq}{\rightarrow} \pi_{i} \widetilde{\mathcal{K}}^{(2)}$ for all $i$ and hence $F^{2} \rightarrow \widetilde{\mathcal{K}}^{(2)}$ is a weak equivalence by the Whitehead theorem. Since $F^{2}$ is an Eilenberg-Mac Lane complex of type $\left(\mathcal{K}_{2}, 2\right)$ by [45, Corollary 8.7], we conclude.

3.2. Cohomology of $\mathcal{K}_{2}$ on a surface. Let us now assume that $X$ is a reduced quasiprojective surface over $k$. Let $\mathcal{K}_{i}(\mathbb{Z} / n)$ denote the Zariski sheaf on $\mathbf{S c h}_{k}$ associated to the presheaf $X \mapsto K_{i}(X, \mathbb{Z} / n)$. Applying the Brown-Gersten spectral sequence (see [14, Theorem 3])

$$
H^{p}\left(X, \pi_{-q} \mathcal{F}\right) \Rightarrow H^{p+q}(X, \mathcal{F})
$$

(3.2) and (3.3), we obtain a commutative diagram of exact sequences

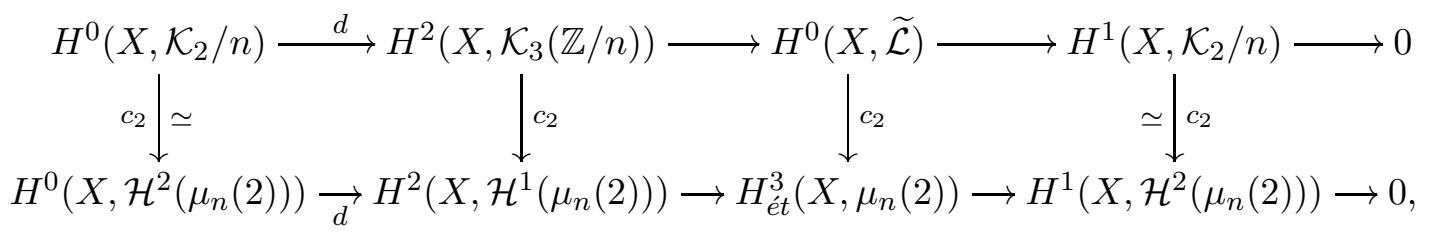

where the vertical arrows are induced by the Chern class map $c_{2, X}$. The bottom row is exact because $\mathcal{H}^{3}\left(\mu_{n}(2)\right)=0$ by [47, Theorem VI.7.2]. The map $H^{0}(X, \widetilde{\mathcal{L}}) \stackrel{c_{2}}{\longrightarrow}$ $H_{\text {ét }}^{3}\left(X, \mu_{n}(2)\right)$ is the one induced by the maps $H^{0}(X, \widetilde{\mathcal{L}}) \stackrel{c_{2}}{\rightarrow} H^{0}\left(X, \Omega \mathcal{E}_{2}\right) \simeq H^{-1}\left(X, \mathcal{E}_{2}\right)=$ $\pi_{1} \mathcal{E}_{2}(X)=H_{e t}^{3}\left(X, \mu_{n}(2)\right)$. The leftmost and the rightmost vertical arrows are isomorphisms by Hoobler's theorem [26, Theorem 3].

Lemma 3.2. There is a functorial map $H^{0}\left(X, \widetilde{\mathcal{L}}^{(2)}\right) \rightarrow \mathbb{H}^{2}\left(X, \mathcal{K}_{2}^{\bullet}\right)$ and a commutative diagram with exact rows

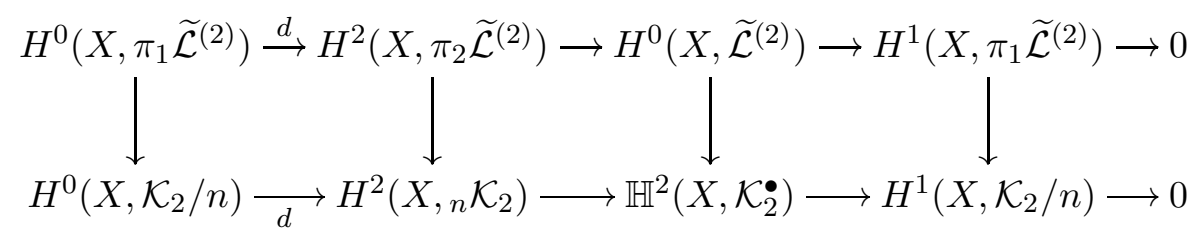

such that all vertical arrows are isomorphisms.

Proof. The bottom exact sequence follows from the exact triangle

$$
{ }_{n} \mathcal{K}_{2} \rightarrow \mathcal{K}_{2}^{\bullet} \rightarrow \mathcal{K}_{2} / n[-1] \rightarrow{ }_{n} \mathcal{K}_{2}[1]
$$


in the derived category of Zariski sheaves of abelian groups on $\mathbf{S c h}_{k} /$ zar. On the other hand, Lemma 3.1 says that $\widetilde{\mathcal{K}}^{(2)}$ is an Eilenberg-Mac Lane complex of the type $\left(\mathcal{K}_{2}, 2\right)$ and there is a homotopy equivalence $\widetilde{\mathcal{L}}^{(2)} \rightarrow \mathcal{K}_{2}^{\bullet}[2]$. This in particular implies that $\pi_{1} \widetilde{\mathcal{L}}^{(2)} \simeq \mathcal{K}_{2} / n, \pi_{2} \widetilde{\mathcal{L}}^{(2)} \simeq{ }_{n} \mathcal{K}_{2}$ and $\pi_{i} \widetilde{\mathcal{L}}^{(2)}=0$ for $i \neq 1,2$. Applying the Brown-Gersten spectral sequence to $H^{0}\left(X, \widetilde{\mathcal{L}}^{(2)}\right)$ and using Lemma 3.1, we conclude the proof. The commutativity follows because both rows are obtained by applying the hypercohomology spectral sequence to the map $\widetilde{\mathcal{L}}^{(2)} \rightarrow \mathcal{K}_{2}^{\bullet}[2]$.

The key step in extending (3.1) to arbitrary surfaces is the following result.

Lemma 3.3. The map $H^{2}\left(X, \mathcal{K}_{3}(\mathbb{Z} / n)\right) \rightarrow H^{2}\left(X,{ }_{n} \mathcal{K}_{2}\right)$, induced by the universal coefficient theorem, has a natural factorization

$$
H^{2}\left(X, \mathcal{K}_{3}(\mathbb{Z} / n)\right) \stackrel{c_{2}}{\rightarrow} H^{2}\left(X, \mathcal{H}^{1}\left(\mu_{n}(2)\right)\right) \stackrel{\nu}{\rightarrow} H^{2}\left(X,{ }_{n} \mathcal{K}_{2}\right)
$$

such that the map $\nu$ is an isomorphism.

Proof. By [3, Lemma 6.2, Variant 6.3], there is a commutative diagram

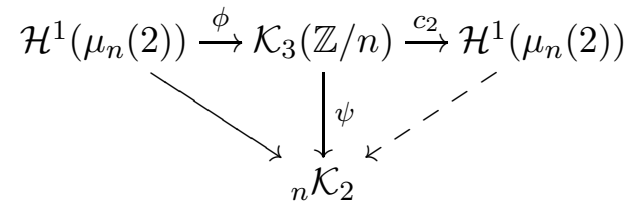

such that $c_{2} \circ \phi=-1$ and the composite $\psi \circ \phi$ is given by $a \mapsto\{a, \zeta\} \in{ }_{n} \mathcal{K}_{2}$ (where $\zeta \in k^{\times}$is a primitive $n^{\text {th }}$ root of unity). This composite map is surjective and an isomorphism on the regular locus of $X$ by [3, Lemma 6.2]. It follows that the induced map $\psi \circ \phi: H^{2}\left(X, \mathcal{H}^{1}\left(\mu_{n}(2)\right)\right) \rightarrow H^{2}\left(X,{ }_{n} \mathcal{K}_{2}\right)$ is an isomorphism (see [52, Lemma 1.3]). We thus have a diagram

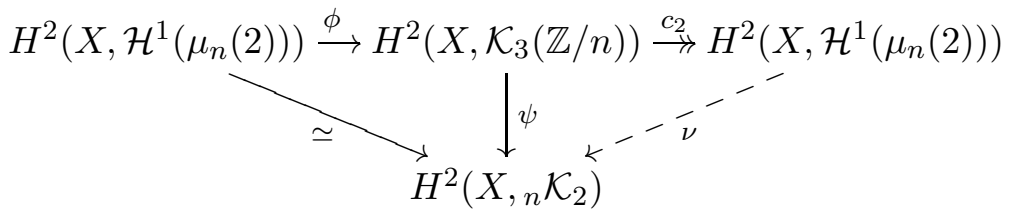

in which the triangle on the left is commutative. To prove the lemma, it is therefore sufficient to show that $\operatorname{Ker}\left(c_{2}\right)=\operatorname{Ker}(\psi)$. Equivalently, $\operatorname{Ker}\left(c_{2}\right) \subseteq \operatorname{Ker}(\psi)$.

We set $\mathcal{F}=\operatorname{Ker}\left(c_{2}\right)$ so that we have a split exact sequence

$$
0 \rightarrow \mathcal{F} \rightarrow \mathcal{K}_{3}(\mathbb{Z} / n) \stackrel{c_{2}}{\rightarrow} \mathcal{H}^{1}\left(\mu_{n}(2)\right) \rightarrow 0 .
$$

By [52, Lemma 1.3], it suffices to show that the composite map $\mathcal{F} \rightarrow \mathcal{K}_{3}(\mathbb{Z} / n) \stackrel{\psi}{\rightarrow}{ }_{n} \mathcal{K}_{2}$ of Zariski sheaves is zero on the smooth locus of $X$. Since this is a local question, it suffices to show that for a regular local ring $A$ which is essentially of finite type over $k$, the map $\operatorname{Ker}\left(c_{2}\right) \rightarrow{ }_{n} K_{2}(A)$ is zero. By the Gersten resolution of $K_{2}(A)$, Bloch-Ogus resolution for $H_{e ́ t}^{1}\left(A, \mu_{n}(2)\right)$ and Gillet's resolution for $K_{3}(A, \mathbb{Z} / n)$ (see [53], [13] and [23]), we can replace $A$ by its fraction field $F$.

We now have a commutative diagram

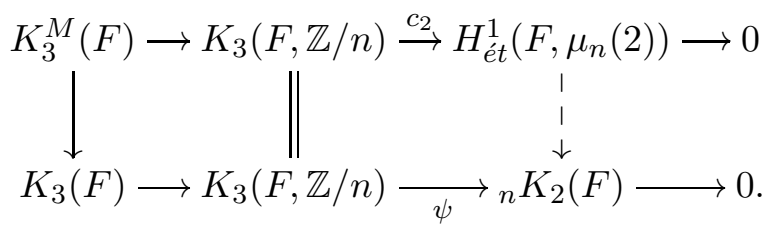


One of the main results of [40] (and also [46]) shows that the top row in (3.11) is exact. Since the bottom row is clearly exact, we get the desired conclusion.

Our first main result of this section is the following extension of (3.1) to surfaces with arbitrary singularities.

Theorem 3.4. Let $X$ be a reduced quasi-projective surface over $k$ and let $n \geq 1$ be an integer prime to $p$. Then, there is a short exact sequence

$$
0 \rightarrow H^{1}\left(X, \mathcal{K}_{2}\right) \otimes_{\mathbb{Z}} \mathbb{Z} / n \rightarrow H_{\text {ét }}^{3}\left(X, \mu_{n}(2)\right) \rightarrow{ }_{n} \mathrm{CH}_{0}(X) \rightarrow 0 .
$$

Proof. It follows from Lemma 3.2 and the map between the Brown-Gersten spectral sequences associated to the morphism of simplicial presheaves $\widetilde{\mathcal{L}} \rightarrow \widetilde{\mathcal{L}}^{(2)}$ that there is a commutative diagram
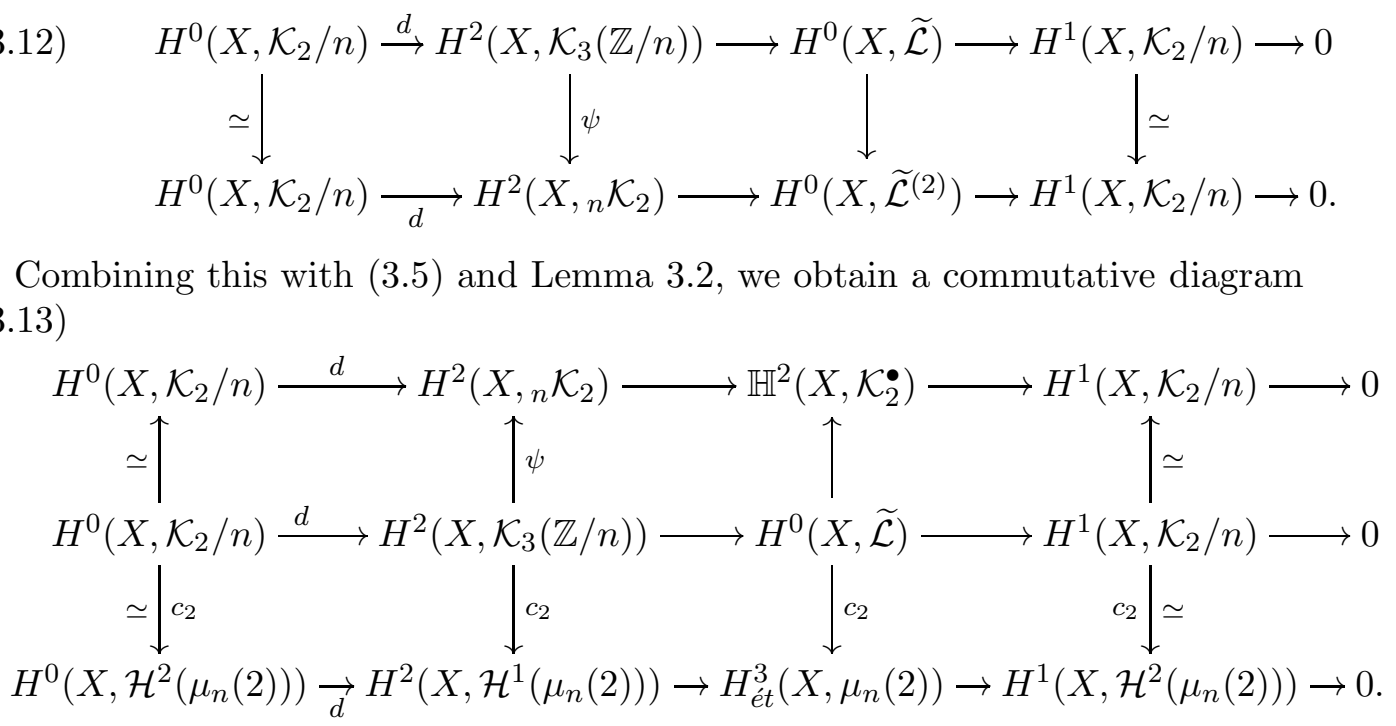

It follows from Lemma 3.3 that $\psi$ and the corresponding vertical arrow downward are surjective with identical kernels. A simple diagram chase shows that the maps $H^{0}(X, \widetilde{\mathcal{L}}) \rightarrow \mathbb{H}^{2}\left(X, \mathcal{K}_{2}^{\bullet}\right)$ and $H^{0}(X, \widetilde{\mathcal{L}}) \rightarrow H_{e ̂ t}^{3}\left(X, \mu_{n}(2)\right)$ are surjective with identical kernels. In particular, there is a natural isomorphism $c_{2, X}: \mathbb{H}^{2}\left(X, \mathcal{K}_{2}^{\bullet}\right) \stackrel{\simeq}{\rightarrow} H_{\text {ét }}^{3}\left(X, \mu_{n}(2)\right)$. Using the exact sequence

$$
0 \rightarrow H^{1}\left(X, \mathcal{K}_{2}\right) \otimes_{\mathbb{Z}} \mathbb{Z} / n \rightarrow \mathbb{H}^{2}\left(X, \mathcal{K}_{2}^{\bullet}\right) \rightarrow{ }_{n} H^{2}\left(X, \mathcal{K}_{2}\right) \rightarrow 0
$$

and the isomorphisms $H^{2}\left(X, \mathcal{K}_{2}\right) \stackrel{\simeq}{\rightarrow} \mathrm{CH}_{0}^{L W}(X) \stackrel{\simeq}{\rightarrow} \mathrm{CH}_{0}(X)$ (see [37, Theorem 7] and [7, Theorem 3.17]), we now conclude the proof.

3.3. Theorem $\mathbf{1 . 1}$ for singular surfaces. As an application of Theorem 3.4, we now prove a version of Theorem 1.1 for singular surfaces. This result was proven for normal projective surfaces in [3, Theorem 7.9]. Let $l \neq p$ be a prime number. Let $X$ be a reduced projective surface over $k$. We shall make no distinction between $\mathrm{CH}_{0}^{L W}(X)$ and $\mathrm{CH}_{0}(X)$ in view of [7, Theorem 3.17].

Lemma 3.5. $H_{e ́ t}^{3}\left(X, \mathbb{Q}_{l} / \mathbb{Z}_{l}(2)\right)$ is divisible by $l^{n}$ for every $n \geq 1$.

Proof. The exact sequence of Theorem 3.4 is compatible with the maps $\mathbb{Z} / l^{n} \rightarrow \mathbb{Z} / l^{n+1}$. Taking the direct limit, we obtain a short sequence

$$
0 \rightarrow H^{1}\left(X, \mathcal{K}_{2}\right) \otimes_{\mathbb{Z}} \mathbb{Q}_{l} / \mathbb{Z}_{l} \rightarrow H_{e ́ t}^{3}\left(X, \mathbb{Q}_{l} / \mathbb{Z}_{l}(2)\right) \stackrel{\tau_{X}}{\longrightarrow} \mathrm{CH}_{0}(X)\{l\} \rightarrow 0 .
$$

The group on the left is divisible. It is known that $\mathrm{CH}_{0}(X)_{\operatorname{deg} 0}$ is generated by the images of the maps $\operatorname{Pic}^{0}(C) \rightarrow \mathrm{CH}_{0}(X)$, where $C \subsetneq X$ is a reduced Cartier curve. 
Since $\operatorname{Pic}^{0}(C)$ is $l^{n}$-divisible, it follows that $\mathrm{CH}_{0}(X)_{\operatorname{deg} 0}$ is $l^{n}$-divisible. In particular, $\mathrm{CH}_{0}(X)\{l\}=\mathrm{CH}_{0}(X)_{\operatorname{deg} 0}\{l\}$ is also $l^{n}$-divisible. The lemma follows.

Theorem 3.6. Given a reduced projective surface $X$ over $k$ and a prime $l \neq p$, the following hold.

(1) $H^{1}\left(X, \mathcal{K}_{2}\right) \otimes_{\mathbb{Z}} \mathbb{Q}_{l} / \mathbb{Z}_{l}=0$.

(2) The map $\tau_{X}: H_{e ́ t}^{3}\left(X, \mathbb{Q}_{l} / \mathbb{Z}_{l}(2)\right) \rightarrow \mathrm{CH}_{0}(X)\{l\}$ is an isomorphism.

Proof. In view of (3.14), the theorem is equivalent to showing that $\tau_{X}$ is injective. To show this, it suffices to prove the stronger assertion that the map $\delta:=\rho_{X}^{\text {semi }} \circ \tau_{X}$ : $H_{e ́ t}^{3}\left(X, \mathbb{Q}_{l} / \mathbb{Z}_{l}(2)\right) \rightarrow \mathrm{CH}_{0}(X)\{l\} \rightarrow J^{2}(X)\{l\}$ is an isomorphism.

In order to prove this, we first prove a stronger version of its surjectivity assertion, namely, that for every $n \geq 1$, the map $H_{e ́ t}^{3}\left(X, \mu_{l^{n}}(2)\right) \rightarrow l^{n} J^{2}(X)$ is surjective. In view of Theorem 3.4, it suffices to show that the map $l^{n} \mathrm{CH}_{0}(X) \rightarrow{ }_{l^{n}} J^{2}(X)$ is surjective. To prove this, we use [42, Theorem 14], which says that we can find a reduced Cartier curve $C \subsetneq X$ (a suitable hypersurface section in a projective embedding) such that $C \cap X_{\text {reg }} \subseteq$ $C_{\text {reg }}$ and the induced map $l^{n} \mathrm{Pic}^{0}(C) \rightarrow l^{n} J^{2}(X)$ is surjective. The commutative diagram

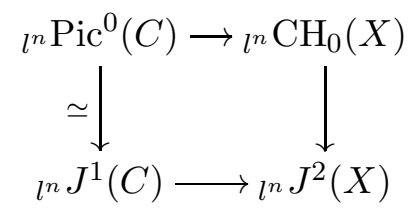

then proves the desired surjectivity where the left vertical arrow is an isomorphism because the $\operatorname{Ker}\left(\operatorname{Pic}^{0}(C) \rightarrow J^{1}(C)\right)$ is unipotent, and hence uniquely $l^{n}$-divisible.

In the rest of the proof, we shall ignore the Tate twist in étale cohomology. Let $\delta_{n}$ denote the composite map $H_{e t}^{3}\left(X, \mu_{l^{n}}(2)\right) \rightarrow{ }_{l^{n}} \mathrm{CH}_{0}(X) \rightarrow{ }_{l^{n}} J^{2}(X)$ so that $\delta=\underset{n}{\lim } \delta_{n}$. Using the above surjectivity, we get a direct system of short exact sequences

$$
0 \rightarrow T_{l^{n}}(X) \rightarrow H_{e ́ t}^{3}\left(X, \mu_{l^{n}}(2)\right) \rightarrow l^{n} J^{2}(X) \rightarrow 0
$$

whose direct limit is the short exact sequence

$$
0 \rightarrow T_{l \infty}(X) \rightarrow H_{\text {ét }}^{3}\left(X, \mathbb{Q}_{l} / \mathbb{Z}_{l}(2)\right) \rightarrow J^{2}(X)\{l\} \rightarrow 0 .
$$

To prove the theorem, we are only left with showing that $T_{l \infty}(X)=0$. Since $H_{\text {ét }}^{3}\left(X, \mathbb{Q}_{l} / \mathbb{Z}_{l}(2)\right) \simeq \underset{n}{\lim } H_{\text {ét }}^{3}\left(X, \mu_{l^{n}}(2)\right) \simeq \underset{n}{\lim _{n}} H_{\text {ét }}^{3}\left(X, \mathbb{Z} / l^{n}\right)$, it follows that $H_{\text {ét }}^{3}\left(X, \mathbb{Q}_{l} / \mathbb{Z}_{l}(2)\right)$ $=H_{e ́ t}^{3}\left(X, \mathbb{Q}_{l} / \mathbb{Z}_{l}(2)\right)\{l\}$. In particular, $T_{l}(X)$ is an $l$-primary torsion group.

We next consider a commutative diagram of short exact sequences

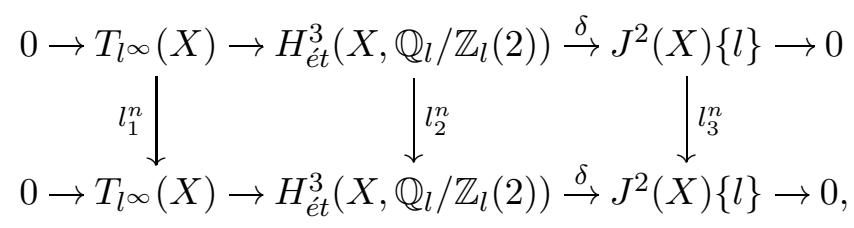

in which the vertical arrows are simply multiplication by $l^{n}$. It follows from Lemma 3.5 that the middle vertical arrow is surjective. We have shown above that $H_{e ́ t}^{3}\left(X, \mu_{l^{n}}(2)\right) \rightarrow$ $l^{n} J^{2}(X)$ is surjective. In particular, the map $\operatorname{ker}\left(l_{2}^{n}\right) \rightarrow \operatorname{Ker}\left(l_{3}^{n}\right)$ is surjective. It follows that $T_{l \infty} \otimes_{\mathbb{Z}} \mathbb{Z} / l^{n}=0$ for every $n \geq 1$. We thus only have to show that $T_{l \infty}$ is finite to finish the proof.

Now, an easy argument that involves dualizing the argument of [4, Theorem 2.5.4] (see [42, Proof of Theorem 15, Claim 2]), shows that there is a positive integer $N_{X}$, depending only on $X$ and not on the integer $n$, such that

$$
\left.\mid H_{e t}^{3}\left(X, \mu_{l^{n}}(2)\right)\right)\left|\leq N_{X} \cdot\right|{ }_{l^{n}} J^{2}(X) \mid .
$$


Combining this with (3.15), it follows that for every $n \geq 1$, either $l^{n} J^{2}(X)=0$ and hence $T_{l^{n}}(X)=0$ or $l^{n} J^{2}(X) \neq 0$ and $\left|T_{l^{n}}(X)\right| \cdot\left|l^{n} J^{2}(X)\right| \leq N_{X} \cdot\left|l_{l^{n}} J^{2}(X)\right|$. In particular, either $T_{l^{n}}(X)=0$ or $\left|T_{l^{n}}(X)\right| \leq N_{X}$ for every $n \geq 1$. But this implies that $T_{l^{\infty}}(X)$ is finite. This completes the proof of the theorem.

3.4. Relation with Bloch's construction. In [10, § 2], Bloch constructed a map $\lambda_{X}: \mathrm{CH}_{0}(X)\{l\} \rightarrow H_{e ́ t}^{2 d-1}\left(X, \mathbb{Q}_{l} / \mathbb{Z}_{l}(2)\right)$ for a smooth projective scheme $X$ of dimension $d \geq 1$ over $k$. We end this section with the following lemma that explains the relation between Bloch's construction and ours when $X$ is a smooth surface.

Lemma 3.7. If $X$ is a smooth projective surface over $k$, then $\tau_{X}: H_{e t}^{3}\left(X, \mathbb{Q}_{l} / \mathbb{Z}_{l}(2)\right) \rightarrow$ $\mathrm{CH}_{0}(X)\{l\}$ coincides with the negative of the inverse of Bloch's map $\lambda_{X}: \mathrm{CH}_{0}(X)\{l\} \rightarrow$ $H_{e t}^{3}\left(X, \mathbb{Q}_{l} / \mathbb{Z}_{l}(2)\right)$.

Proof. To prove the lemma, we have to go back to the construction of $\tau_{X}: H_{e ́ t}^{3}\left(X, \mu_{n}(2)\right) \rightarrow$ ${ }_{l^{n}} \mathrm{CH}_{0}(X)$ in $\S 3.2$ for $n \in k^{\times}$. Since $X$ is smooth, there is an isomorphism of Zariski sheaves $\mathcal{O}_{X}^{\times} / n \stackrel{\simeq}{\rightarrow} \mathcal{H}^{1}\left(\mu_{n}(2)\right) \stackrel{\simeq}{\rightarrow}{ }_{n} \mathcal{K}_{2}$ by [즈. Lemma 6.2]. Since $H^{2}\left(X, \mathcal{O}_{X}^{\times}\right)=0$, we see that the top cohomology of all these sheaves vanish.

This implies that the map $\mathbb{H}^{2}\left(X, \mathcal{K}_{2}^{\bullet}\right) \rightarrow{ }_{n} H^{2}\left(X, \mathcal{K}_{2}\right)$ is simply the composite $\mathbb{H}^{2}\left(X, \mathcal{K}_{2}^{\bullet}\right)$ $\stackrel{\simeq}{\rightarrow} H^{1}\left(X, \mathcal{K}_{2} / n\right) \rightarrow H^{2}\left(X,{ }_{n} \mathcal{K}_{2}\right) \rightarrow{ }_{n} H^{2}\left(X, \mathcal{K}_{2}\right)$. Moreover, there is a diagram

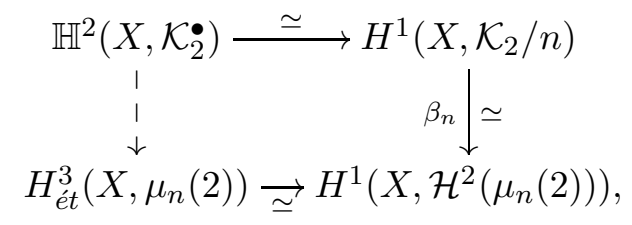

where the right vertical arrow is the Galois symbol map. This induces a unique isomorphism $\alpha_{n}: H_{e ́ t}^{3}\left(X, \mu_{n}(2)\right) \stackrel{\simeq}{\rightarrow} \mathbb{H}^{2}\left(X, \mathcal{K}_{2}^{\bullet}\right)$. One checks from (3.13) that $\tau_{X}$ is simply the composite

$$
H_{e t}^{3}\left(X, \mu_{n}(2)\right) \stackrel{\alpha_{n}}{\longrightarrow} \mathbb{H}^{2}\left(X, \mathcal{K}_{2}^{\bullet}\right) \stackrel{\simeq}{\rightarrow} H^{1}\left(X, \mathcal{K}_{2} / n\right) \stackrel{\delta_{n}}{\longrightarrow}{ }_{n} H^{2}\left(X, \mathcal{K}_{2}\right) .
$$

We also have a commutative diagram

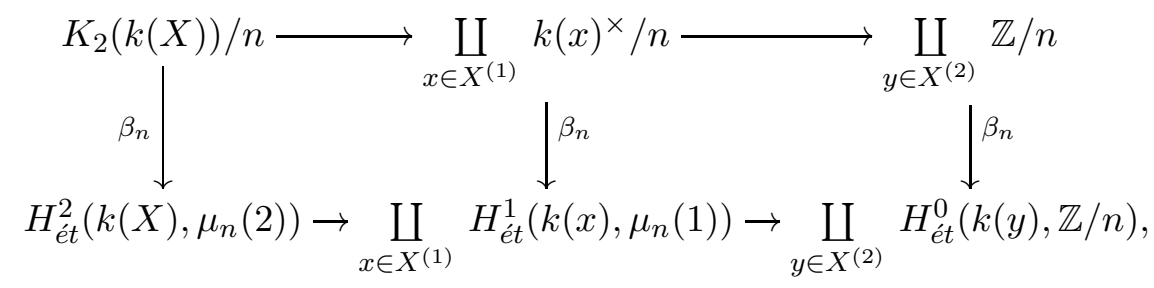

where all vertical arrows are the Galois symbols and are isomorphisms.

Since the Gersten resolution is universally exact (see [53] and [24]), the middle cohomology of the top row is $H^{1}\left(X, \mathcal{K}_{2} / n\right)$ and the Bloch-Ogus resolution (see [13]) shows that the middle cohomology of the bottom row is $H^{1}\left(X, \mathcal{H}^{2}\left(\mu_{n}(2)\right)\right)$. The isomorphism $\beta_{n}$ in (3.18) is induced by the vertical arrows of (3.20).

For a fixed prime $l \neq p$, the map $\delta_{l^{m}}$ in (3.19) becomes an isomorphism on the limit over $m \geq 1$. Assuming this isomorphism, it follows from (3.19) that $\tau_{X}=\underset{m}{\lim } \alpha_{l^{m}}$. On the other hand, it follows from Bloch's construction in [10, $\S 2]$ that $\lambda_{X}=\underset{m}{\lim } \beta_{l^{m}}$. Since each $\alpha_{n}$ (with $n \in k^{\times}$) is the inverse of $\beta_{n}$ by definition, the lemma follows. 


\section{ROITMAN'S TORSION FOR SEPARABLY WEAKLY NORMAL SURFACES}

The Roitman torsion theorem says that for a smooth projective scheme of dimension $d \geq 1$ over $k$, the Abel-Jacobi map $\rho_{X}: \mathrm{CH}_{0}(X)_{\operatorname{deg} 0} \rightarrow J^{d}(X)$ is an isomorphism on the torsion subgroups. This theorem was extended to normal projective schemes in [34]. However, when $X$ has arbitrary singularities, this theorem is known only for the torsion prime-to- $p$ (see [9] and [42]). In this section, we extend the Roitman torsion theorem to separably weakly normal (see below for definition) surfaces. Later in this text, we shall prove a suitable generalization of this theorem in higher dimension. This generalization will be used to prove Theorem 1.2 .

4.1. Separably weakly normal schemes. The weak normality is a singularity type of schemes, which in characteristic $p>0$, is closely related to various $F$-singularities. These $F$-singularities are naturally encountered while running the minimal model program in positive characteristic. Most of the $F$-regularity conditions imply weak normality. In characteristic zero, weak normality coincides with the more familiar notion of semi-normality. In this text, we shall study the Chow group of 0-cycles on certain singular schemes whose singularities are closely related to weak normality in positive characteristic.

Let $A$ be a reduced commutative Noetherian ring. Let $B$ be the integral closure of $A$ in its total quotient ring. Recall from [43] that the semi-normalization of $A$ is the largest among the subrings $A^{\prime}$ of $B$ containing $A$ such that

(1) $\forall x \in \operatorname{Spec}(A)$, there exists exactly one $x^{\prime} \in \operatorname{Spec}\left(A^{\prime}\right)$ over $x$ and

(2) the canonical homomorphism $k(x) \rightarrow k\left(x^{\prime}\right)$ is an isomorphism.

The weak normalization of $A$ is the largest among the subrings $A^{\prime}$ of $B$ containing $A$ such that

(1) $\forall x \in \operatorname{Spec}(A)$, there exists exactly one $x^{\prime} \in \operatorname{Spec}\left(A^{\prime}\right)$ over $x$ and

(2) the field extension $k(x) \rightarrow k\left(x^{\prime}\right)$ is finite purely inseparable.

We let $A_{s} \subset B$ and $A_{w} \subset B$ denote the semi-normalization and weak normalization of $A$, respectively. One says that $A$ is semi-normal (resp. weakly normal) if $A=A_{s}$ (resp. $A=A_{w}$ ). It is clear from the above definition that $A \subset A_{s} \subset A_{w} \subset B$. Moreover, $A_{s}=A_{w}$ if $A$ is a $\mathbb{Q}$-algebra. To get a more geometric understanding of these singularities, we make the following definition.

Definition 4.1. Let $k$ be a field and let $R$ be a $k$-algebra which is finite as a $k$-vector space. We shall say that $R$ is weakly separable over $k$ if it is reduced and either char $(k)=$ 0 , or $\operatorname{char}(k)>0$ and there is no inclusion of rings $k \subsetneq K \subset R$ such that $K$ is a purely inseparable field extension of $k$.

Recall that a commutative Noetherian ring $A$ is called an $S_{2}$ ring if for every prime ideal $\mathfrak{p}$ of $A$, one has $\operatorname{depth}\left(A_{\mathfrak{p}}\right) \geq \min (\mathrm{ht}(\mathfrak{p}), 2)$. It follows easily from this definition that a Cohen-Macaulay ring is $S_{2}$.

Proposition 4.2. Let $A$ be reduced commutative Noetherian ring. Let $B$ be the integral closure of $A$ in its total quotient ring and let $I \subset B$ be the largest ideal which is contained in $A$. Assume that $B$ is a finite $A$-module. Then the following hold.

(1) If $A$ is semi-normal, then $B / I$ is reduced. If $A$ is $S_{2}$, the converse also holds.

(2) If $A$ is weakly normal, then $B / I$ is reduced and the inclusion map $A / I \rightarrow B / I$ is generically weakly separable. If $A$ is $S_{2}$, the converse also holds.

Proof. If $A$ is semi-normal, then $B / I$ is reduced by [58, Lemma 1.3]. Suppose now that $A$ is $S_{2}$ and $B / I$ is reduced. By [25, Theorem 2.6], it suffices to show that $A_{\mathfrak{p}}$ is semi-normal for every height one prime ideal $\mathfrak{p}$ in $A$. 
Let $\mathfrak{p} \subset A$ be a prime ideal of height one. Let $\left\{x_{1}, \cdots, x_{r}\right\} \subset B$ be a subset whose image in $B / A$ generates it as an $A$-module. We now note that $\left(A_{\mathfrak{p}}: B_{\mathfrak{p}}\right)=(A: B)_{\mathfrak{p}}$, where $I:=(A: B)=\{a \in A \mid a B \subset A\}=\operatorname{ann}(B / A)$. The first equality uses the fact that $B / A$ is a finite $A$-module so that $I=\bigcap_{i=1}^{r}\left(A: x_{i}\right)$ and $\left(J \cap J^{\prime}\right)_{\mathfrak{p}}=J_{\mathfrak{p}} \cap J_{\mathfrak{p}}^{\prime}$ (see [44, Ex. 4.8]). Since $B_{\mathfrak{p}} / I_{\mathfrak{p}}=(B / I)_{\mathfrak{p}}$ is reduced (by our assumption) and $A_{\mathfrak{p}}$ is 1-dimensional, it follows from [6, Proposition 7.2] and [58, Theorem 3.6] that $A_{\mathfrak{p}}$ is semi-normal. This proves (1).

Suppose now that $A$ is weakly normal. Then it is clearly semi-normal. In particular, $B / I$ is reduced by (1). We now show that $A / I \hookrightarrow B / I$ is generically weakly separable. Note that as $A$ is reduced and generically weakly normal, $I$ must have height at least one in $A$. Let $\mathfrak{p}$ be a minimal prime of $I$ in $A$ and let $k(\mathfrak{p})$ be its residue field. Then $\mathfrak{p} A_{\mathfrak{p}}=\mathfrak{p} B_{\mathfrak{p}}$ is the Jacobson radical of $B_{\mathfrak{p}}$ and hence $B_{\mathfrak{p}} / \mathfrak{p} B_{\mathfrak{p}}$ is a product of finite field extensions of $k(\mathfrak{p})$.

We consider the commutative diagram

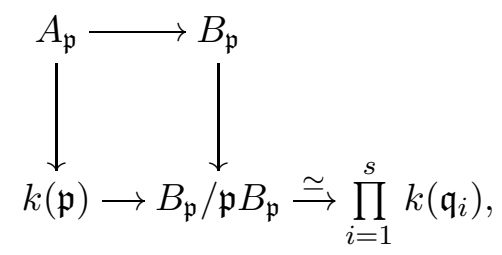

in which the square is Cartesian, the horizontal arrows are injective finite morphisms and the vertical arrows are surjective. Since $A_{\mathfrak{p}}$ is weakly normal (see [43, Theorem IV.3]) and $B_{\mathfrak{p}}$ is its integral closure, it means that $A_{\mathfrak{p}}$ is weakly normal in $B_{\mathfrak{p}}$. We conclude from 62, Proposition 3] that $k(\mathfrak{p})$ is weakly normal in $\prod_{i=1}^{s} k\left(\mathfrak{q}_{i}\right)$. We now apply [62, Lemma 2] to deduce that the lower horizontal arrow in (4.1) is weakly separable, as desired.

Conversely, suppose that $A$ is $S_{2}$ and the inclusion $A / I \hookrightarrow B / I$ is generically weakly separable map of reduced schemes. By [43, Corollary IV. 4], it suffices to show that $A_{\mathfrak{p}}$ is weakly normal for every height one prime ideal $\mathfrak{p}$ in $A$.

Let $\mathfrak{p} \subset A$ be a prime ideal of height one. Since $I_{\mathfrak{p}}=\left(A_{\mathfrak{p}}: B_{\mathfrak{p}}\right)$ as shown above, it follows that $A_{\mathfrak{p}}=B_{\mathfrak{p}}$ (hence $A_{\mathfrak{p}}$ is weakly normal) if $I \not \subset \mathfrak{p}$. We can therefore assume that $I \subset \mathfrak{p}$. Since $A$ is generically normal (and hence weakly normal), $I$ must have height at least one in $A$. It follows that $\mathfrak{p}$ must be a minimal prime ideal of $I$.

It follows from (1) that $A_{\mathfrak{p}}$ is semi-normal. In particular, $I_{\mathfrak{p}}=\mathfrak{p} A_{\mathfrak{p}}=\mathfrak{p} B_{\mathfrak{p}}$. Furthermore, $\mathfrak{p} B_{\mathfrak{p}}$ is the Jacobson radical of $B_{\mathfrak{p}}$ such that $B_{\mathfrak{p}} / \mathfrak{p} B_{\mathfrak{p}}$ is a finite product of finite field extensions of $k(\mathfrak{p})$. This gives rise to a Cartesian square of rings as in (4.1). Our assumption says that the lower horizontal arrow in (4.1) is weakly separable. We conclude again from [62, Lemma 2, Proposition 3] that $A_{\mathfrak{p}}$ is weakly normal. This proves (2).

Example 4.3. Using Proposition 4.2, we can construct many examples of semi-normal rings which are not weakly normal. Let $k$ be perfect field of characteristic $p>0$ and consider the Cartesian square of rings

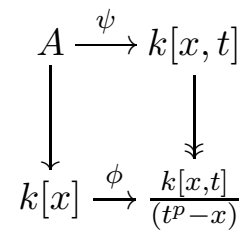

in which the right vertical arrow is the canonical surjection and $\phi$ is the canonical inclusion. In particular, one has $A=\left\{(f(x), g(x, t)) \mid f\left(t^{p}\right)=g\left(t^{p}, t\right)\right\}$. Since $\phi$ is a finite purely inseparable map of reduced rings which is not an isomorphism, it follows that $\psi$ 
is not an isomorphism. It follows from Proposition 4.2 that $A$ is semi-normal but not weakly normal.

Example 4.4. We now provide an example of a weakly normal $S_{2}$ ring $A$ with normalization $B$ and reduced conductor $I$ such that the map $A / I \rightarrow B / I$ is not generically separable. Let $k$ be a perfect field of characteristic $p>0$ and consider the Cartesian square

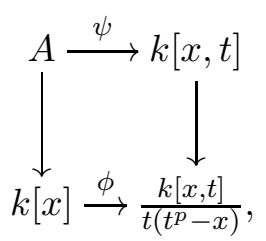

where the right vertical arrow is the canonical quotient map and the lower horizontal arrow is given by $\phi(x)=x$. One can easily check (e.g., use Eisenstein's criterion) that $t^{p}-x$ is an irreducible polynomial in $k[x, t]$. If we let $\mathfrak{q}_{1}=(t)$ and $\mathfrak{q}_{2}=\left(t^{p}-x\right)$, then we see that $k(x) \rightarrow k\left(\mathfrak{q}_{1}\right)$ is an isomorphism and $k(x) \rightarrow k\left(\mathfrak{q}_{2}\right) \stackrel{\simeq}{\rightarrow} k\left(x^{1 / p}\right)$ is purely inseparable. In particular, $\phi$ induces a weakly separable map between the function fields. It follows from [62, Proposition 3] that $A$ is weakly normal. We just saw however that $\phi$ is not generically separable.

Note also that the kernels of the vertical arrows in (4.3) are isomorphic and the kernel on the right is a principal ideal generated by $f(x, t)=t\left(t^{p}-x\right)$. Since $f(x, t)$ a nonzero-divisor in $k[x, t]$ and lies in $A$, it must be a non-zero-divisor in $A$. Since $k[x]$ is Cohen-Macaulay, it follows that $A$ is Cohen-Macaulay too (see [44, Theorem 17.3]). In particular, it is $S_{2}$.

Motivated by Example 4.4, we make the following definition.

Definition 4.5. Let $A$ be a commutative reduced Noetherian ring with finite normalization $B$. Let $I \subset B$ be the largest ideal lying in $A$. We shall say that $A$ is separably weakly normal if $B / I$ is reduced and the induced map $A / I \rightarrow B / I$ is generically separable. We shall say that a Noetherian scheme $X$ is (separably) weakly normal (resp. semi-normal) if the coordinate ring of every affine open in $X$ is (separably) weakly normal (resp. semi-normal).

It follows from Proposition 4.2 that if $A$ is $S_{2}$ and separably weakly normal, then it is weakly normal. On the other hand, Example 4.4 shows that a weakly normal $S_{2}$ ring may not be separably weakly normal. For $\mathbb{Q}$-algebras, semi-normality implies separably weak normality by Proposition 4.2. The three singularity types coincide for $\mathbb{Q}$-algebras which are $S_{2}$. Our goal in the rest of this text is to study the torsion in the Chow group of 0-cycles on schemes which are separably weakly normal.

Remark 4.6. Since we only deal with schemes which are separably weakly normal in the rest of this text, the reader can, in principle, only read Definition 4.5 in $\S 4.1$ and move to the next subsection. Our discussion of semi-normality and weak normality is primarily meant to motivate the reader to Definition 4.5, and to give a comparison of various non-normal singularity types which are closely related yet different.

We end this subsection by recalling the notion of conducting ideals and conducting subschemes. Let $A$ be a reduced commutative Noetherian ring and let $B$ be a subring of the integral closure of $A$ in its total quotient ring such that $A \subset B$. Assume that $B$ is a finite $A$-module. Recall that an ideal $I \subset A$ is called a conducting ideal for the inclusion $A \subset B$ if $I=I B$. It is clear from this definition that $I \subset(A: B)$. Furthermore, one knows that $(A: B)$ is the largest conducting ideal for $A \subset B$ (e.g., see [27, Ex. 2.11]). 
If $X$ is a reduced Noetherian scheme and $f: X^{\prime} \rightarrow X$ is a finite birational map, then a closed subscheme $Y \subset X$ is called a conducting subscheme if $\mathcal{I}_{Y} \subset \mathcal{O}_{X}$ is a sheaf of conducting ideals for the inclusion of sheaves of rings $\mathcal{O}_{X} \subset f_{*}\left(\mathcal{O}_{X^{\prime}}\right)$.

4.2. The torsion theorem for separably weakly normal surfaces. For the remaining part of this section, we shall identify the two Chow groups $\mathrm{CH}_{0}^{L W}(X)$ and $\mathrm{CH}_{0}(X)$ for curves and surfaces using [7, Theorem 3.17]. To prove the Roitman torsion theorem for a separably weakly normal projective surface, we need the following excision result for our Chow group. We fix an algebraically closed field $k$.

Lemma 4.7. Let $X$ be a reduced quasi-projective separably weakly normal surface over $k$ and let $f: \bar{X} \rightarrow X$ denote the normalization map. Let $Y \subset X$ denote the smallest conducting closed subscheme with $\bar{Y}=Y \times_{X} \bar{X}$. Then there is an exact sequence

$$
S K_{1}(\bar{X}) \oplus S K_{1}(Y) \rightarrow S K_{1}(\bar{Y}) \rightarrow \mathrm{CH}_{0}(X) \rightarrow \mathrm{CH}_{0}(\bar{X}) \rightarrow 0 .
$$

Proof. We have a commutative diagram of relative and birelative $K$-theory exact sequences:

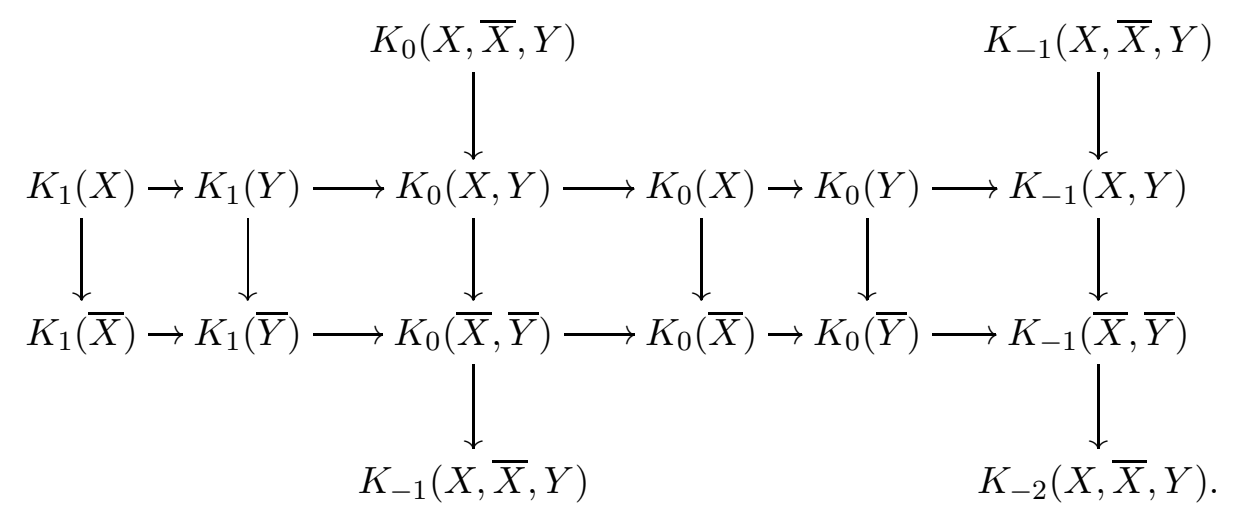

Using the Thomason-Trobaugh spectral sequence and the results of Bass 5 that the sheaves $\mathcal{K}_{i,(X, \bar{X}, Y)}$ on $Y$ vanish for $i \leq 0$, we see that $K_{i}(X, \bar{X}, Y)=0$ for $i \leq-1$. It also follows from the spectral sequence that $K_{0}(X, \bar{X}, Y) \simeq H^{1}\left(Y, \mathcal{K}_{1,(X, \bar{X}, Y)}\right)$. It follows from [21, Theorem 0.2] that $H^{1}\left(Y, \mathcal{K}_{1,(X, \bar{X}, Y)}\right) \simeq H^{1}\left(X, \mathcal{I}_{Y} / \mathcal{I}_{Y}^{2} \otimes_{\mathcal{O}_{Y}} \Omega_{\bar{Y} / Y}^{1}\right)$. Since $X$ is separably weakly normal, it follows from Definition 4.5 that the map $\bar{Y} \rightarrow Y$ is a finite generically étale map of reduced schemes. Hence $\Omega \bar{Y}_{Y}{ }_{Y}$ has 0 -dimensional support and we conclude that $H^{1}\left(X, \mathcal{I}_{Y} / \mathcal{I}_{Y}^{2} \otimes_{\mathcal{O}_{Y}} \Omega_{\bar{Y} / Y}^{1}\right)=0$. In particular, $K_{0}(X, \bar{X}, Y)=0$.

A diagram chase in (4.5) shows that there is an exact sequence

$$
K_{1}(\bar{X}) \oplus K_{1}(Y) \rightarrow K_{1}(\bar{Y}) \rightarrow \widetilde{K}_{0}(X) \rightarrow \widetilde{K}_{0}(\bar{X}) \oplus \widetilde{K}_{0}(Y) \rightarrow \widetilde{K}_{0}(\bar{Y})
$$

where $\widetilde{K}_{0}(-)=\operatorname{Ker}\left(K_{0}(-) \rightarrow H^{0}(-, \mathbb{Z})\right)$. Using the map of sheaves $\mathcal{O}_{X}^{\times} \rightarrow f_{*}\left(\mathcal{O}_{\bar{X}}^{\times}\right)$, this exact sequence maps to a similar unit-Pic exact sequence

$$
U(\bar{X}) \oplus U(Y) \rightarrow U(\bar{Y}) \rightarrow \operatorname{Pic}(X) \rightarrow \operatorname{Pic}(\bar{X}) \oplus \operatorname{Pic}(Y) \rightarrow \operatorname{Pic}(\bar{Y}) .
$$

Taking the kernels and using the Levine's formula $S K_{0}(Z):=\operatorname{Ker}\left(\widetilde{K}_{0}(Z) \rightarrow \operatorname{Pic}(Z)\right) \simeq$ $H^{2}\left(Z, \mathcal{K}_{2}\right) \simeq \mathrm{CH}_{0}(Z)$ for a reduced surface $Z$, we get (4.4).

Lemma 4.8. Let $Y$ be a reduced curve over $k$ and let $r \geq 0$ denote the number of irreducible components of $Y$ which are projective over $k$. If $Y$ is affine, then $S K_{1}(Y)$ is uniquely divisible. If $Y$ is projective, then $S K_{1}(Y)$ is divisible, $S K_{1}(Y)\{l\} \simeq\left(\mathbb{Q}_{l} / \mathbb{Z}_{l}\right)^{r}$ for a prime $l \neq p$ and $S K_{1}(Y)\{p\}=0$. 
Proof. It follows from [3, Theorem 5.3] that $S K_{1}(Y) \simeq\left(k^{\times}\right)^{r} \oplus V$, where $V$ is uniquely divisible. The lemma follows directly from this isomorphism.

Theorem 4.9. Let $X$ be a reduced projective separably weakly normal surface over an algebraically closed field $k$ of exponential characteristic $p$. Then, $A^{2}(X)$ is a semi-abelian variety and the Abel-Jacobi map $\rho_{X}: \mathrm{CH}_{0}(X)_{\operatorname{deg} 0} \rightarrow A^{2}(X)$ is an isomorphism on the torsion subgroups.

Proof. We can find a finite collection of reduced complete intersection Cartier curves $\left\{C_{1}, \cdots, C_{r}\right\}$ on $X$ such that the induced map of algebraic groups $\prod_{i=1}^{r} \operatorname{Pic}^{0}\left(C_{i}\right) \rightarrow A^{2}(X)$ is surjective (see, for instance, [18, (7.1), p. 657]). In characteristic zero, we can further assume by [15, Corollary 2.5] that each of these curves is weakly normal. Since a surjective morphism of smooth connected algebraic groups restricts to a surjective map on their unipotent and semi-abelian parts and since $\operatorname{Pic}^{0}(C)$ is semi-abelian if $C$ is a weakly normal curve (easy to check), it follows that $A^{2}(X)$ is a semi-abelian variety in characteristic zero.

In characteristic $p \geq 2$, the surjections $\prod_{i=1}^{r} \operatorname{Pic}^{0}\left(C_{i}\right) \rightarrow A^{2}(X) \rightarrow J^{2}(X)$ and 31, Lemma 2.7] together imply that the induced maps $\left(\mathrm{CH}^{2}(X)_{\operatorname{deg} 0}\right)_{\text {tors }} \rightarrow A^{2}(X)_{\text {tors }} \rightarrow$ $J^{2}(X)_{\text {tors }}$ are also surjective. Since $A_{\text {unip }}^{2}(X)$ is a $p$-primary torsion group of bounded exponent, the theorem will follow if we prove, in any characteristic, that the composite map $\rho_{X}^{\text {semi }}: \mathrm{CH}_{0}(X)_{\operatorname{deg} 0} \rightarrow A^{2}(X) \rightarrow J^{2}(X)$ is injective on the torsion subgroup.

It follows from [42, Theorem 15] that the map $\mathrm{CH}_{0}(X)_{\operatorname{deg} 0}\{l\} \rightarrow J^{2}(X)\{l\}$ is an isomorphism for every prime $l \neq p$. This also follows immediately from Theorem 3.4 and the proof of Theorem 3.6. We can thus assume that $p \geq 2$ and $l=p$.

Let $f: \bar{X} \rightarrow X$ be the normalization and consider the commutative diagram

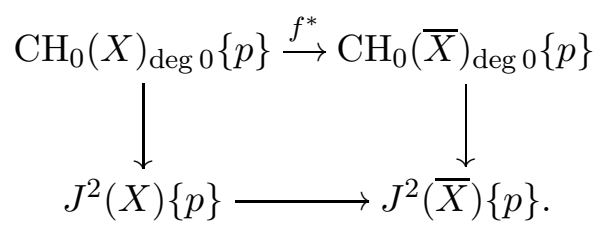

The right vertical arrow is an isomorphism by [34, Theorem 1.6]. Thus, it suffices to prove the stronger assertion that the map $f^{*}: \mathrm{CH}_{0}(X)\{p\} \rightarrow \mathrm{CH}_{0}(\bar{X})\{p\}$ is an isomorphism. It is enough to show that $A:=\operatorname{Ker}\left(\mathrm{CH}_{0}(X) \rightarrow \mathrm{CH}_{0}(\bar{X})\right)$ is uniquely $p$-divisible.

It follows from Lemma 4.7 that there is an exact sequence

$$
S K_{1}(\bar{X}) \oplus S K_{1}(Y) \rightarrow S K_{1}(\bar{Y}) \rightarrow A \rightarrow 0 .
$$

Following the notations of Lemma 4.7, it follows from Lemma 4.8 that $S K_{1}(Y)$ and $S K_{1}(\bar{Y})$ are uniquely $p$-divisible. Furthermore, it follows from [31, Theorem 5.6] that $S K_{1}(\bar{X}) \otimes \mathbb{Q}_{p} / \mathbb{Z}_{p}=0$. An elementary argument now shows that $A$ must be uniquely $p$-divisible. This finishes the proof.

Corollary 4.10. Let $X$ be a reduced projective separably weakly normal surface over $\overline{\mathbb{F}}_{p}$. Then $\mathrm{CH}_{0}(X)_{\operatorname{deg} 0}$ is finite-dimensional. That is, the Abel-Jacobi map $\rho_{X}: \mathrm{CH}_{0}(X)_{\operatorname{deg} 0} \rightarrow$ $A^{2}(X)$ is an isomorphism.

Proof. In view of Theorem 4.9, it suffices to show that for any reduced projective scheme $X$ of dimension $d \geq 1$ over $\overline{\mathbb{F}}_{p}$, the group $\mathrm{CH}_{0}^{L W}(X)_{\operatorname{deg} 0}$ is a torsion abelian group.

Given $\alpha \in \mathrm{CH}_{0}^{L W}(X)_{\operatorname{deg} 0}$, we can find a reduced Cartier curve $C \subseteq X$ such that $\alpha$ lies in the image of the push-forward map $\mathrm{Pic}^{0}(C) \rightarrow \mathrm{CH}_{0}^{L W}(X)_{\operatorname{deg} 0}$. It is therefore enough 
to show that $\operatorname{Pic}^{0}(C)$ is torsion. But this is a special case of the more general fact that $G\left(\overline{\mathbb{F}}_{p}\right)$ is torsion whenever $G$ is a smooth commutative algebraic group over $\overline{\mathbb{F}}_{p}$.

\section{BLOCH'S TORSION THEOREM FOR 0-CYCLES WITH MODULUS}

We continue with our assumption that $k$ is algebraically closed with exponential characteristic $p$. Let $X$ be a smooth projective scheme of dimension $d \geq 1$ over $k$ and let $D \subset X$ be an effective Cartier divisor on $X$. We shall prove Theorem 1.1] in this section.

5.1. Relative étale cohomology. Given an étale sheaf $\mathcal{F}$ on $\mathbf{S c h}_{k}$ and a finite map $f: Y \rightarrow X$ in $\operatorname{Sch}_{k}$, let $\mathcal{F}_{(X, Y)}:=\operatorname{Cone}\left(\left.\mathcal{F}\right|_{X} \rightarrow f_{*}\left(\left.\mathcal{F}\right|_{Y}\right)\right)[-1]$ be the chain complex of étale sheaves on $X$. The exactness of $f_{*}$ on étale sheaves implies that there is a long exact sequence of étale (hyper)cohomology groups

$$
0 \rightarrow H_{\text {ét }}^{0}\left(X, \mathcal{F}_{(X, Y)}\right) \rightarrow H_{e ́ t}^{0}(X, \mathcal{F}) \rightarrow H_{e ́ t}^{0}(Y, \mathcal{F}) \rightarrow H_{e ́ t}^{1}\left(X, \mathcal{F}_{(X, Y)}\right) \rightarrow \cdots .
$$

If $Y \hookrightarrow X$ is a closed immersion with complement $j: U \hookrightarrow X$, then one checks immediately from the above definition that $H_{e t}^{*}\left(X, \mathcal{F}_{(X, Y)}\right)$ is canonically isomorphic to $H_{e t}^{*}\left(X, j_{!}\left(\left.\mathcal{F}\right|_{U}\right)\right)$. We conclude that if $X$ is projective over $k$ and $Y \hookrightarrow X$ is closed, then $H_{e t t}^{*}\left(X, \mathcal{F}_{(X, Y)}\right)$ is same as the étale cohomology with compact support $H_{c, e ́ t}^{*}(X \backslash Y, \mathcal{F})$ of $X \backslash Y$.

Let us now consider an abstract blow-up diagram in $\mathbf{S c h}_{k}$ :

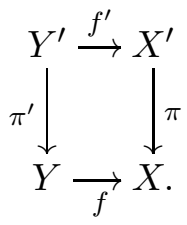

This is a Cartesian square in which the horizontal arrows are closed immersions, $\pi$ is a proper morphism such that $X^{\prime} \backslash Y^{\prime} \stackrel{\simeq}{\rightarrow} X \backslash Y$. The proper base change theorem for the étale cohomology implies that for a torsion constructible étale sheaf $\mathcal{F}$ on $\mathbf{S c h}_{k}$, the cohomology groups $H_{e t}^{*}(-, \mathcal{F})$ satisfy the $c d h$-descent. In particular, the canonical map

$$
\pi^{*}: H_{e ́ t}^{i}\left(X, \mathcal{F}_{(X, Y)}\right) \rightarrow H_{e ́ t}^{i}\left(X^{\prime}, \mathcal{F}_{\left(X^{\prime}, Y^{\prime}\right)}\right)
$$

is an isomorphism for every $i \geq 0$. We shall write the relative étale cohomology groups $H_{e t}^{*}\left(X, \mathcal{F}_{(X, Y)}\right)$ for a closed immersion $Y \hookrightarrow X$ as $H_{e t}^{*}(X \mid Y, \mathcal{F})$.

5.2. A weak Lefschetz type theorem for the double. We now come back to our situation of $X$ being a smooth projective scheme of dimension $d \geq 1$ over $k$ and $D \subset X$ an effective Cartier divisor. Let $\left\{E_{1}, \cdots, E_{r}\right\}$ be the set of irreducible components of $D_{\text {red. }}$ If $d \geq 3$, we choose, as in $\S\left[2.5\right.$, a closed embedding $X \hookrightarrow \mathbb{P}_{k}^{N}$ and a smooth hypersurface section $\tau: Y=X \cap H_{1} \hookrightarrow X$ such that $Y$ is not contained in $D$ and $Y \cap E_{i}$ is integral for every $1 \leq i \leq r$. We set $F=D \cap Y$. We shall use these notations throughout the rest of this section.

Given a prime-to- $p$ integer $n$, it is clear from the definition of the relative étale cohomology and its $c d h$-descent (see $\S$ 5.1) associated to the Cartesian square (2.1) (see Lemma 2.21) that the pull-back maps via the closed immersions $\iota_{ \pm}: X \hookrightarrow S_{X}$ induce, for each $i \geq 0$, a split exact sequence of étale cohomology

$$
0 \rightarrow H_{e ́ t}^{i}\left(X \mid D, \mu_{n}(j)\right) \stackrel{p_{+, *}}{\longrightarrow} H_{e ́ t}^{i}\left(S_{X}, \mu_{n}(j)\right) \stackrel{\iota_{-}^{*}}{\longrightarrow} H_{e ́ t}^{i}\left(X, \mu_{n}(j)\right) \rightarrow 0 .
$$

Here, the splitting of $\iota_{-}^{*}$ is given by the pull-back $\Delta^{*}: H_{e t}^{i}\left(X, \mu_{n}(j)\right) \rightarrow H_{e t t}^{i}\left(S_{X}, \mu_{n}(j)\right)$.

Let us next recall from Gabber's construction [19] of the Gysin morphism for étale cohomology (see, also [50, Definition 2.1]) that the regular closed embeddings $\tau: Y \hookrightarrow X$ and $\tau_{1}: S_{Y} \hookrightarrow S_{X}$ induce Gysin morphisms $\tau_{*}: H_{e ́ t}^{i}\left(Y, \mu_{n}(j)\right) \rightarrow H_{e ́ t}^{i+2}\left(X, \mu_{n}(j+1)\right)$ and 
$\tau_{1, *}: H_{e ́ t}^{i}\left(S_{Y}, \mu_{n}(j)\right) \rightarrow H_{e ́ t}^{i+2}\left(S_{X}, \mu_{n}(j+1)\right)$ for $i \geq 0$. Furthermore, it follows from the Cartesian square (2.9) and [50, Corollary 2.12] (see also [19, Proposition 1.1.3]) that the pull-back via the closed immersions $\iota_{ \pm}: X \hookrightarrow S_{X}$ induces a commutative diagram

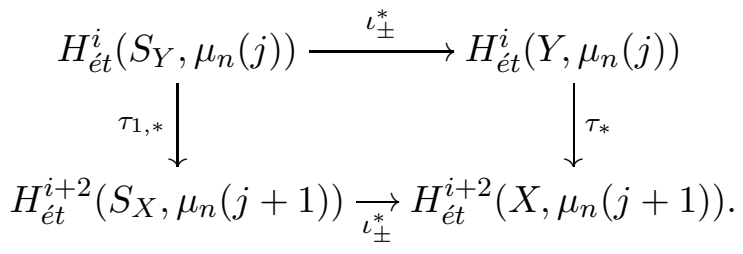

Lemma 5.1. If $d \geq 3$, then the Gysin maps $\tau_{*}: H_{e ́ t}^{2 d-3}\left(Y, \mu_{n}(d-1)\right) \rightarrow H_{e ́ t}^{2 d-1}\left(X, \mu_{n}(d)\right)$ and $\tau_{1, *}: H_{e ́ t}^{2 d-3}\left(S_{Y}, \mu_{n}(d-1)\right) \rightarrow H_{e ́ t}^{2 d-1}\left(S_{X}, \mu_{n}(d)\right)$ are isomorphisms.

Proof. The first isomorphism is a well known consequence of the weak Lefschetz theorem for étale cohomology. The main problem is to prove the second isomorphism. Since $k$ is algebraically closed, we shall replace $\mu_{n}$ by the constant sheaf $\Lambda=\mathbb{Z} / n$.

Recall from [17, § 2] that the line bundle $\mathcal{O}_{X}(Y)$ (which we shall write in short as $\mathcal{O}(Y)$ ) on $X$ has a canonical class $[\mathcal{O}(Y)] \in H_{Y, e ́ t}^{1}\left(X, \mathbb{G}_{m}\right)$ and its image via the boundary map $H_{Y, e ́ t}^{1}\left(X, \mathbb{G}_{m}\right) \rightarrow H_{Y, e ́ t}^{2}(X, \Lambda)$ is Deligne's localized Chern class $c_{1}(Y)$. Here, $H_{Y, e ́ t}^{*}(X,-)$ denotes the étale cohomology with support in $Y$. On the level of the derived category $D^{+}(Y, \Lambda)$, this Chern class is given in terms of the map $c_{1}(Y): \Lambda \rightarrow \tau^{!} \Lambda(1)[2]$. Using this Chern class, Gabber's Gysin morphism $\tau_{*}: H_{e ́ t}^{*}(Y, \Lambda(j)) \rightarrow H_{e ́ t}^{*+2}(X, \Lambda(j+1))$ is the one induced on the cohomology by the composite map $\tau_{*}: \tau_{*}\left(\Lambda_{Y}\right) \rightarrow \tau_{*} \tau^{!}\left(\Lambda_{Y}(1)[2]\right) \rightarrow$ $\Lambda_{X}(1)[2]$ in $D^{+}(X, \Lambda)$.

Corresponding to the Cartesian square (2.9), we have $\pi^{*}\left(\mathcal{O}\left(S_{Y}\right)\right)=\mathcal{O}(Y \amalg Y)$ and hence we have a commutative diagram

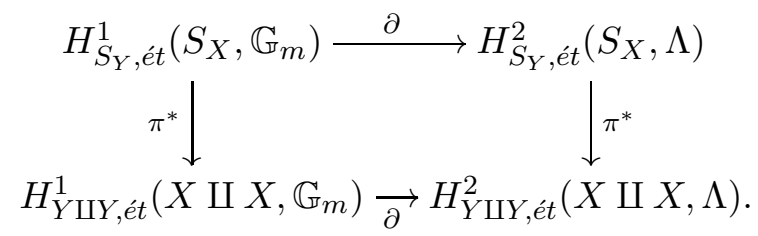

We thus have commutative diagrams

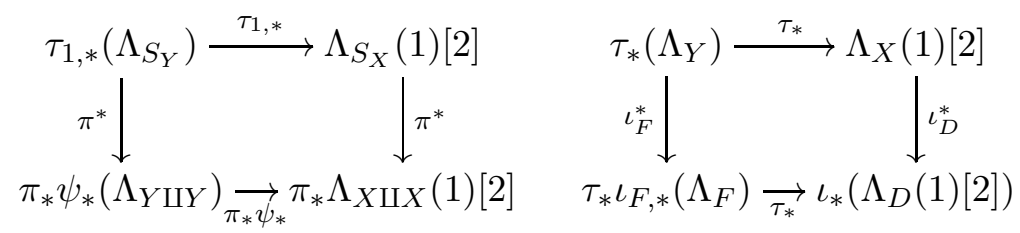

in $D^{+}\left(S_{X}, \Lambda\right)$ and $D^{+}(X, \Lambda)$.

We next observe that the canonical map $\Lambda_{X \amalg X} \rightarrow \Lambda_{X} \oplus \Lambda_{X}$, induced by the inclusions of the two components, is an isomorphism. We thus have a sequence of maps $\Lambda_{S_{X}} \stackrel{\pi^{*}}{\longrightarrow}$ $\pi_{*}\left(\Lambda_{X \amalg X}\right) \simeq \Lambda_{X_{+}} \oplus \Lambda_{X_{-}} \rightarrow \Lambda_{D}$, where the last map is the difference of two restrictions $\Lambda_{X_{ \pm}} \rightarrow \iota_{*}\left(\Lambda_{D}\right)$. Furthermore, it is easy to check that the sequence

$$
0 \rightarrow \Lambda_{S_{X}}(j) \stackrel{\pi^{*}}{\rightarrow} \pi_{*}\left(\Lambda_{X \amalg X}(j)\right) \rightarrow \Lambda_{D}(j) \rightarrow 0
$$


is exact. A combination of this with (5.7) yields a commutative diagram of exact triangles in $D^{+}\left(S_{X}, \Lambda\right)$ :

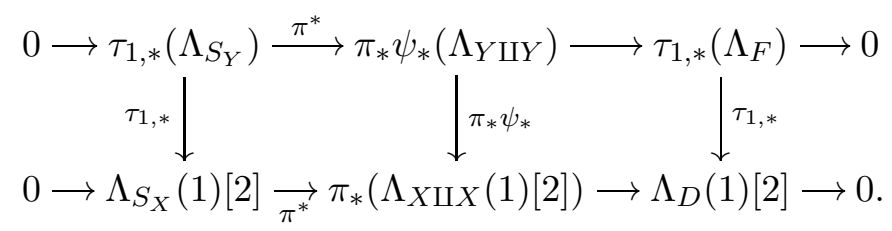

Since all the underlying maps in (2.9) are finite, we obtain a commutative diagram of long exact sequence of cohomology groups

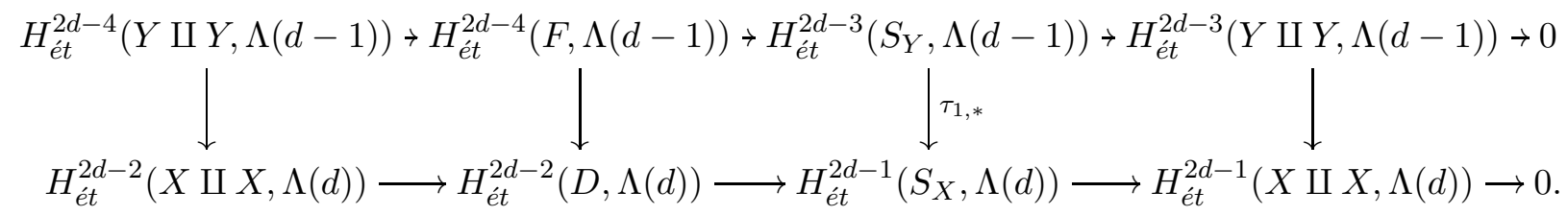

Since $d \geq 3$, it follows from the weak Lefschetz theorem for étale cohomology (see 47, Theorem VI.7.1]) that the vertical arrow on the left end of (5.9) is surjective and the one on the right end is an isomorphism. We next note that the inclusion $F=D \cap H_{1} \hookrightarrow D$ induces a bijection between the irreducible components of $F$ and $D$ by our choice of the hypersurface $H_{1}$ (see $\S 2.5$ ). It follows from this, together with the isomorphism $H_{e ́ t}^{2 d-2}(D, \Lambda) \stackrel{\simeq}{\rightarrow} H_{e t}^{2 d-2}\left(D^{N}, \Lambda\right)$ and [47, Lemma VI.11.3], that the second vertical arrow from the left in (5.9) is an isomorphism. A diagram chase now shows that $\tau_{1, *}$ is an isomorphism. This proves the lemma.

Remark 5.2. We should warn here that Lemma 5.1 proves an analogue of the weak Lefschetz theorem only for a specific étale cohomology group. We do not expect this to be true for other cohomology groups in general and it will depend critically on $D$.

5.3. The torsion in Chow group with modulus and relative étale cohomology. Let $D \subset X$ be an effective Cartier divisor on a smooth scheme $X$ as above. Recall from [7, $\S 4,5]$ that there are maps $p_{ \pm, *}: \mathrm{CH}_{0}(X \mid D) \rightarrow \mathrm{CH}_{0}\left(S_{X}\right)$ and $\iota_{ \pm}^{*}: \mathrm{CH}_{0}\left(S_{X}\right) \rightarrow \mathrm{CH}_{0}(X)$, where $p_{ \pm, *}([x])=\iota_{ \pm, *}([x])$ and $\iota_{ \pm}^{*}$ is induced by the projection map $\mathcal{Z}_{0}\left(S_{X} \backslash D\right)=$ $\mathcal{Z}_{0}\left(X_{+} \backslash D\right) \oplus \mathcal{Z}_{0}\left(X_{-} \backslash D\right) \rightarrow \mathcal{Z}_{0}\left(X_{ \pm} \backslash D\right)$. It follows at once from this description of the projection maps $\iota_{ \pm}^{*}$ that there is a commutative diagram

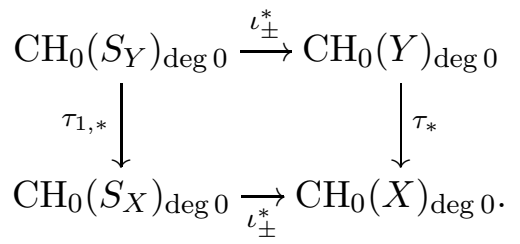

We shall use the following decomposition theorem from [7, Theorem 7.1].

Theorem 5.3. The projection map $\Delta: S_{X} \rightarrow X$ induces a flat pull-back $\Delta^{*}: \mathrm{CH}_{0}(X) \rightarrow$ $\mathrm{CH}_{0}\left(S_{X}\right)$ such that $\iota_{ \pm}^{*} \circ \Delta^{*}=\mathrm{Id}_{\mathrm{CH}_{0}(X)}$. Moreover, there is a split exact sequence

$$
0 \rightarrow \mathrm{CH}_{0}(X \mid D) \stackrel{p_{+, *}}{\longrightarrow} \mathrm{CH}_{0}\left(S_{X}\right) \stackrel{\iota_{-}^{*}}{\longrightarrow} \mathrm{CH}_{0}(X) \rightarrow 0 .
$$

Theorem 5.4. Let $X$ be a smooth projective scheme of dimension $d \geq 1$ over an algebraically closed field $k$ of exponential characteristic $p$. Then for any prime $l \neq p$, there is an isomorphism

$$
\lambda_{X \mid D}: \mathrm{CH}_{0}(X \mid D)\{l\} \stackrel{\simeq}{\rightarrow} H_{e ́ t}^{2 d-1}\left(X \mid D, \mathbb{Q}_{l} / \mathbb{Z}_{l}(d)\right) .
$$


Proof. If $D=\emptyset$, we take $\lambda_{X \mid D}$ to be the isomorphism $\lambda_{X}: \mathrm{CH}_{0}(X)\{l\} \stackrel{\simeq}{\rightarrow} H_{e ́ t}^{2 d-1}\left(X, \mathbb{Q}_{l} / \mathbb{Z}_{l}(d)\right)$ given by Bloch [10, $\S 2]$.

We now assume $D \neq \emptyset$ and let $S_{X}$ be the double of $X$ along $D$. We shall first prove by induction on $d$ that there exists an isomorphism

$$
\lambda_{S_{X}}: \mathrm{CH}_{0}\left(S_{X}\right)\{l\} \stackrel{\simeq}{\rightarrow} H_{\text {ét }}^{2 d-1}\left(S_{X}, \mathbb{Q}_{l} / \mathbb{Z}_{l}(d)\right)
$$

such that $\iota_{ \pm}^{*} \circ \lambda_{S_{X}}=\lambda_{X} \circ \iota_{ \pm}^{*}$.

When $d=1$, it follows easily from the Kummer sequence and [41, Proposition 1.4] that there is a natural isomorphism $H_{e ́ t}^{1}\left(C, \mu_{n}(1)\right) \stackrel{\simeq}{\rightarrow}{ }_{n} \mathrm{CH}_{0}(C)$ for any reduced curve $C$ over $k$ and any integer $n \geq 1$ prime to $p$. The naturality of this isomorphism proves our assertion. Note that this isomorphism coincides with that of Bloch when $C$ is smooth, as one directly checks (or see [10, Proposition 3.6]).

We next assume $d=2$. In this case, we have shown in Theorem 3.4 that there is a homomorphism $\tau_{Y}: H_{e ̂ t}^{3}\left(Y, \mu_{n}(2)\right) \rightarrow{ }_{n} \mathrm{CH}_{0}(Y)$ for any reduced quasi-projective surface $Y$ over $k$ and any integer $n$ prime to $p$. We claim that the diagram

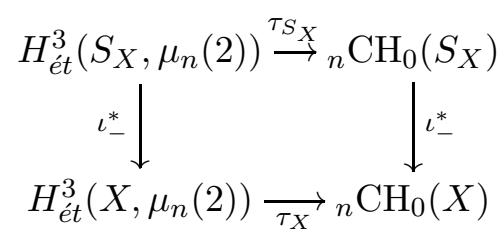

commutes.

To prove this, recall from the construction of $\tau_{Y}$ in $\S 3$ that there are isomorphisms $H_{\text {ét }}^{3}\left(Y, \mu_{n}(2)\right) \stackrel{\simeq}{\rightarrow} \mathbb{H}^{2}\left(Y, \mathcal{K}_{2}^{\bullet}\right)$ and $\mathrm{CH}_{0}(Y) \stackrel{\simeq}{\rightarrow} \mathrm{CH}_{0}^{L W}(X) \stackrel{\simeq}{\rightarrow} H^{2}\left(Y, \mathcal{K}_{2}\right)$ (see [7, Theorem 3.17]) which are clearly functorial for the normalization map $Y^{N} \rightarrow Y$. Since $X_{ \pm}$ are two disjoint components of the normalization $S_{X}^{N}$, we see that these isomorphisms are functorial for the inclusions $\iota_{ \pm}: X_{ \pm} \hookrightarrow S_{X}$.

For a surface $Y$, the map $\tau_{Y}$ is then the natural map $\mathbb{H}^{2}\left(Y, \mathcal{K}_{2}^{\bullet}\right) \rightarrow{ }_{n} H^{2}\left(Y, \mathcal{K}_{2}\right)$ obtained via the exact sequence $\mathbb{H}^{2}\left(Y, \mathcal{K}_{2}^{\bullet}\right) \rightarrow H^{2}\left(Y, \mathcal{K}_{2}\right) \stackrel{n}{\rightarrow} H^{2}\left(Y, \mathcal{K}_{2}\right)$. The commutativity of (5.13) then follows immediately from the naturality of the complex of Zariski sheaves on $\mathcal{K}_{2}[-1] \rightarrow \mathcal{K}_{2}^{\bullet} \rightarrow \mathcal{K}_{2}$ on $\mathbf{S c h}_{k}$. This proves the claim.

Theorem 3.6 says that $\tau_{Y}: H_{e t}^{3}\left(Y, \mathbb{Q}_{l} / \mathbb{Z}_{l}(2)\right) \stackrel{\simeq}{\rightarrow} \mathrm{CH}_{0}(Y)\{l\}$ is an isomorphism on the limit for every prime $l \neq p$. We let $\lambda_{Y}: \mathrm{CH}_{0}(Y)\{l\} \rightarrow H_{e ́ t}^{3}\left(Y, \mathbb{Q}_{l} / \mathbb{Z}_{l}(2)\right)$ be the negative of the inverse of this isomorphism. When $Y$ is smooth over $k$, it follows from Lemma 3.7 that $\lambda_{Y}$ agrees with Bloch's isomorphism. It follows then from (5.13) that

$$
\iota_{ \pm}^{*} \circ \lambda_{S_{X}}=\lambda_{X} \circ \iota_{ \pm}^{*} \text {. }
$$

We now assume $\operatorname{dim}(X) \geq 3$. We choose a closed embedding $X \hookrightarrow \mathbb{P}_{k}^{N}$ and hypersurfaces $H_{1}, \cdots, H_{d-2}$ as in (2.7). We continue to use the notations of Proposition 2.3 . We set $Y=X_{1}:=X \cap H_{1}$ and assume by induction that there is an isomorphism $\lambda_{S_{Y}}: \mathrm{CH}_{0}\left(S_{Y}\right)\{l\} \stackrel{\simeq}{\rightarrow} H_{e ́ t}^{2 d-3}\left(S_{Y}, \mathbb{Q}_{l} / \mathbb{Z}_{l}(d-1)\right)$ such that the diagram

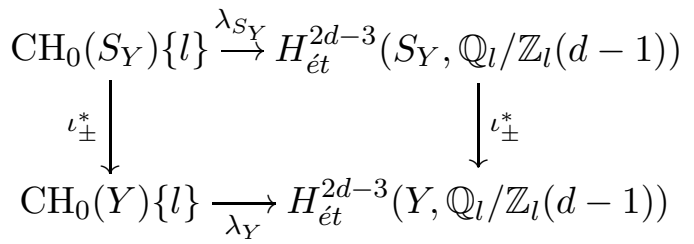

commutes. 
We now consider the diagram

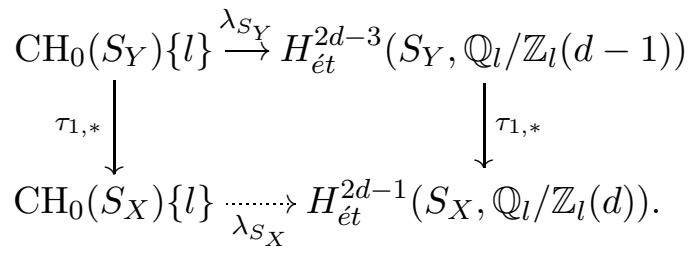

It follows from Proposition 2.3 that the left vertical arrow is an isomorphism and Lemma 5.1 says that the right vertical arrow is an isomorphism. Since $\lambda_{S_{Y}}$ is an isomorphism too, it follows that there is a unique isomorphism $\lambda_{S_{X}}: \mathrm{CH}_{0}\left(S_{X}\right)\{l\} \stackrel{\simeq}{\longrightarrow}$ $H_{e ́ t}^{2 d-1}\left(S_{X}, \mathbb{Q}_{l} / \mathbb{Z}_{l}(d)\right)$ such that (5.16) commutes.

We have to check that $\iota_{ \pm}^{*} \circ \lambda_{S_{X}}=\lambda_{X} \circ \iota_{ \pm}^{*}$. For this, we consider the diagram

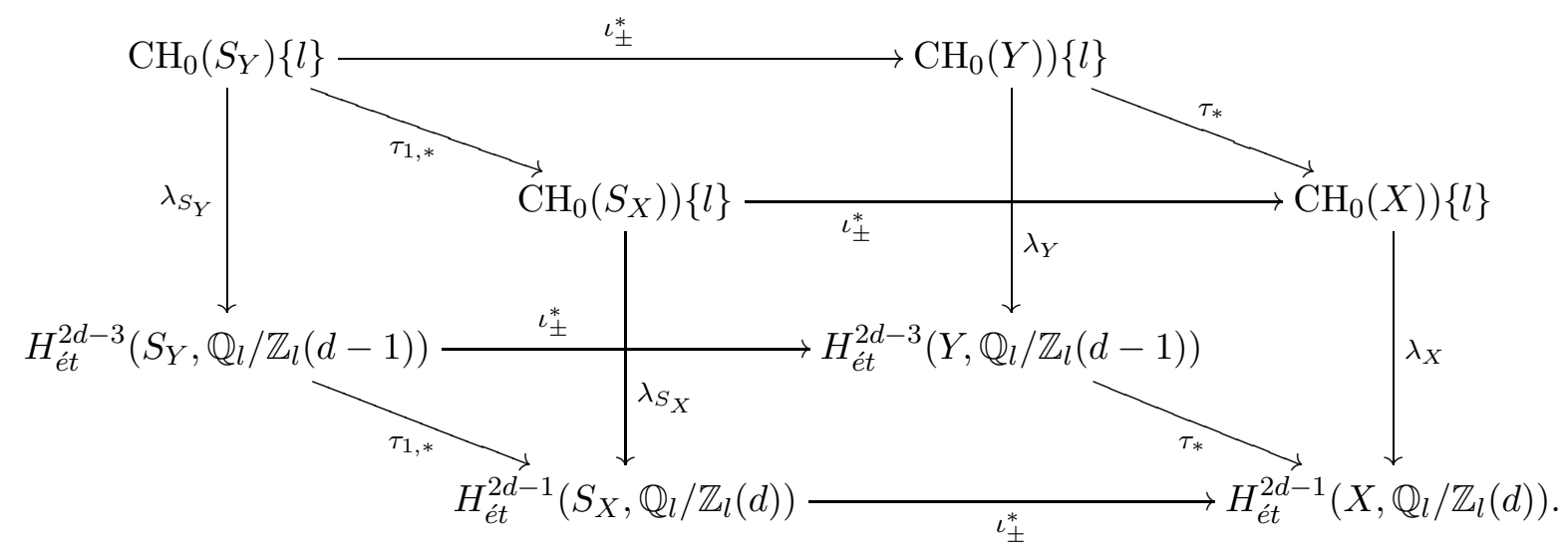

We need to show that the front face of this cube commutes. Since $\tau_{1, *}: \mathrm{CH}_{0}\left(S_{Y}\right)\{l\} \rightarrow$ $\mathrm{CH}_{0}\left(S_{X}\right)\{l\}$ is an isomorphism, it suffices to show that all other faces of the cube commute. The top face commutes by (5.10) and the bottom face commutes by (5.5). The left face commutes by (5.16) and the right face commutes by [10, Proposition 3.3]. Finally, the back face commutes by (5.15) and we are done.

To finish the proof of the theorem, we use (5.4) and Theorem 5.3 and consider the diagram of split exact sequences:

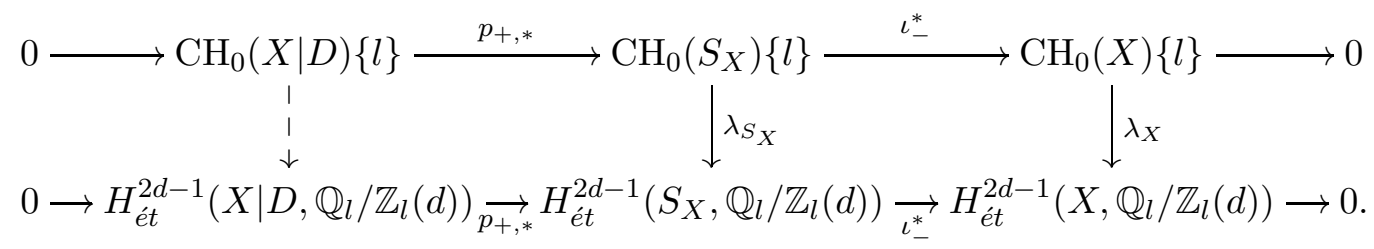

It follows from (5.12) that the square on the right is commutative. Moreover, the maps $\lambda_{S_{X}}$ and $\lambda_{X}$ are both isomorphisms. We conclude that there is an isomorphism $\lambda_{X \mid D}: \mathrm{CH}_{0}(X \mid D)\{l\} \stackrel{\simeq}{\rightarrow} H_{e ́ t}^{2 d-1}\left(X \mid D, \mathbb{Q}_{l} / \mathbb{Z}_{l}(d)\right)$.

5.4. Applications. We now deduce two applications of Theorem 5.4. Since the étale cohomology of $\mu_{n}(j)$ is nil-invariant whenever $(n, p)=1$, it follows from (5.1) that $H_{\text {ét }}^{*}\left(X \mid D, \mu_{n}(j)\right) \stackrel{\simeq}{\rightarrow} H_{e t}^{*}\left(X \mid D_{\text {red }}, \mu_{n}(j)\right)$. Using Theorem 5.4, we therefore obtain the following result about the prime-to- $p$ torsion in the Chow group with modulus.

Theorem 5.5. Let $X$ be a smooth projective scheme of dimension $d \geq 1$ over $k$ let $D \subset X$ be an effective Cartier divisor. Then, the restriction map ${ }_{n} \mathrm{CH}_{0}(X \mid D) \rightarrow$ ${ }_{n} \mathrm{CH}_{0}\left(X \mid D_{\text {red }}\right)$ is an isomorphism for every integer $n$ prime to $p$. 
Our second application is the following extension of Theorem 3.6 to the cohomology of relative $K_{2}$-sheaf on a smooth surface. Recall that for a closed immersion $Y \hookrightarrow X$ in $\mathbf{S c h}_{k}$, the relative $K$-theory sheaf $\mathcal{K}_{i,(X, Y)}$ is the Zariski sheaf on $X$ associated to the presheaf $U \mapsto K_{i}(U, Y \cap U)$.

Theorem 5.6. Let $X$ be a smooth projective surface over an algebraically closed field $k$ of exponential characteristic $p$ and let $l \neq p$ be a prime. Let $D \subset X$ be an effective Cartier divisor. Then, the following hold.

(1) $H^{1}\left(X, \mathcal{K}_{2,(X, D)}\right) \otimes_{\mathbb{Z}} \mathbb{Q}_{l} / \mathbb{Z}_{l}=0$.

(2) $H^{2}\left(X, \mathcal{K}_{2,(X, D)}\right)\{l\} \simeq H_{\text {ét }}^{3}\left(X \mid D, \mathbb{Q}_{l} / \mathbb{Z}_{l}(2)\right)$.

Proof. It follows from Theorem 5.4 that $H_{\text {ét }}^{3}\left(X \mid D, \mathbb{Q}_{l} / \mathbb{Z}_{l}(2)\right) \simeq \mathrm{CH}_{0}(X \mid D)\{l\}$. On the other hand, a combination of [7, Theorem 1.7] and [30, Lemma 2.1] shows that there is a canonical isomorphism $\mathrm{CH}_{0}(X \mid D) \stackrel{\simeq}{\rightarrow} H^{2}\left(X, \mathcal{K}_{2,(X, D)}\right)$. This proves $(2)$.

To prove (1), we consider the following commutative diagram for any integer $n \in k^{\times}$.

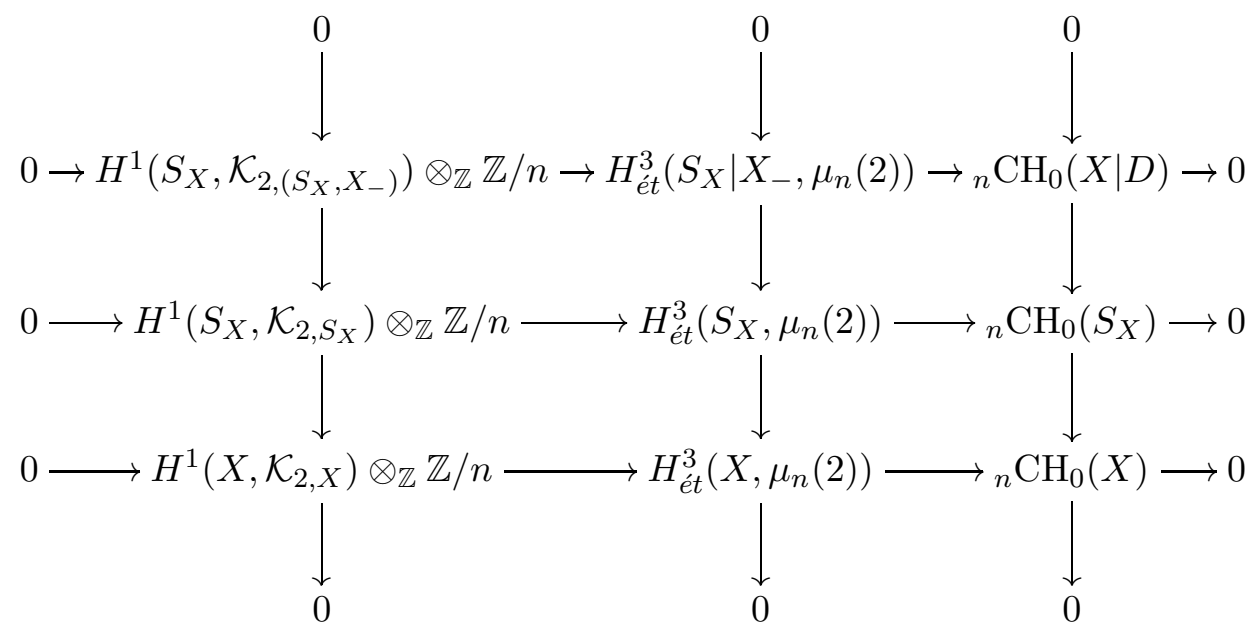

The columns on the left and in the middle are exact by the splitting $\Delta \circ \iota_{-}=\operatorname{Id}_{X}$ in (2.1). The column on the right is exact by Theorem 5.3. The middle and the bottom rows are exact by Theorem 3.4. It follows that the top row is also exact. Using the isomorphism $\iota_{+}^{*}: H_{e ́ t}^{3}\left(S_{X} \mid X_{-}, \mu_{n}(2)\right) \stackrel{\simeq}{\rightarrow} H_{e ̂ t}^{3}\left(X \mid D, \mu_{n}(2)\right)$ (see (5.30) ) and taking the direct limit of the terms in (5.18) with respect to the direct system $\left\{\mathbb{Z} / l^{n}\right\}_{n}$, we obtain a short exact sequence

$$
0 \rightarrow H^{1}\left(S_{X}, \mathcal{K}_{2,\left(S_{X}, X_{-}\right)}\right) \otimes_{\mathbb{Z}} \mathbb{Q}_{l} / \mathbb{Z}_{l} \rightarrow H_{e ́ t}^{3}\left(X \mid D, \mathbb{Q}_{l} / \mathbb{Z}_{l}(2)\right) \rightarrow \mathrm{CH}_{0}(X \mid D)\{l\} \rightarrow 0
$$

Using (5.19) Theorem 5.4, we conclude that $H^{1}\left(S_{X}, \mathcal{K}_{2,\left(S_{X}, X_{-}\right)}\right) \otimes_{\mathbb{Z}} \mathbb{Q}_{l} / \mathbb{Z}_{l}=0$ for every prime $l \neq p$. To finish the proof, it suffices now to show that the pull-back map $\iota_{+}^{*}: H^{1}\left(S_{X}, \mathcal{K}_{2,\left(S_{X}, X_{-}\right)}\right) \rightarrow H^{1}\left(X, \mathcal{K}_{2,(X, D)}\right)$ is surjective.

Given an open subset $W \subset D$, let $U=S_{X} \backslash(D \backslash W)$ be the open subset of $S_{X}$. Let $\mathcal{K}_{i,\left(S_{X}, X_{-}, D\right)}$ be the Zariski sheaf on $D$ associated to the presheaf $W \mapsto K_{i}\left(U, X_{+} \cap\right.$ $\left.U, X_{-} \cap U\right)=\operatorname{hofib}\left(\left(K\left(U, X_{-} \cap U\right) \stackrel{i_{+}^{*}}{\longrightarrow} K\left(X_{+} \cap U, D \cap U\right)\right)\right.$ (see [52, Proposition A.5]). There is an exact sequence of $K$-theory sheaves

$$
\nu_{*}\left(\mathcal{K}_{2,\left(S_{X}, X_{-}, D\right)}\right) \rightarrow \mathcal{K}_{2,\left(S_{X}, X_{-}\right)} \rightarrow \mathcal{K}_{2,\left(X_{+}, D\right)} \rightarrow \nu_{*}\left(\mathcal{K}_{1,\left(S_{X}, X_{-}, D\right)}\right),
$$

where $\nu: D \hookrightarrow S_{X}$ is the inclusion. We have $\mathcal{K}_{1,\left(S_{X}, X_{-}, D\right)}=\mathcal{I}_{D} / \mathcal{I}_{D}^{2} \otimes_{D} \Omega_{D / X}^{1}$ by 21 , Theorem 1.1] and the latter term is zero. We thus get an exact sequence

$$
\nu_{*}\left(\mathcal{K}_{2,\left(S_{X}, X_{-}, D\right)}\right) \rightarrow \mathcal{K}_{2,\left(S_{X}, X_{-}\right)} \rightarrow \mathcal{K}_{2,\left(X_{+}, D\right)} \rightarrow 0 .
$$


Since $H^{2}\left(S_{X}, \iota_{*}\left(\mathcal{K}_{2,\left(S_{X}, X_{-}, D\right)}\right)=H^{2}\left(D, \mathcal{K}_{2,\left(S_{X}, X_{-}, D\right)}\right)=0\right.$, we get $H^{1}\left(X, \mathcal{K}_{2,\left(S_{X}, X_{-}\right)}\right) \rightarrow$ $H^{1}\left(X, \mathcal{K}_{2,\left(X_{+}, D\right)}\right) \simeq H^{1}\left(X, \mathcal{K}_{2,(X, D)}\right)$

\section{RoITMAN'S TORSION THEOREM FOR 0-CYCLES With MODULUS}

In this section, we prove the Roitman torsion theorem (Theorem 1.2) for the Chow group of 0-cycles with modulus. We shall deduce this result by proving a more general Roitman torsion theorem for singular varieties which are obtained by joining two smooth schemes along a common reduced Cartier divisor. As before, we assume the base field $k$ to be algebraically closed of exponential characteristic $p$.

6.1. Join of two smooth schemes along a common divisor. Let $X_{+}$and $X_{-}$ be two smooth connected quasi-projective schemes of dimension $d \geq 1$ over $k$ and let $X_{+} \stackrel{i_{+}}{\longleftarrow} D \stackrel{i_{-}}{\hookrightarrow} X_{-}$be two closed embeddings such that $D$ is a reduced effective Cartier divisor on each $X_{ \pm}$via these embeddings. Let $X$ be the quasi-projective scheme over $k$ such that the square

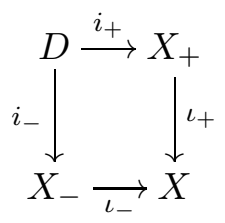

is co-Cartesian in $\mathbf{S c h}_{k}$.

It is easy to check that all arrows in (6.1) are closed immersions and $X$ is a reduced scheme with irreducible components $\left\{X_{+}, X_{-}\right\}$with $X_{\text {sing }}=D$. In particular, the canonical map $\pi=\iota_{+} \amalg \iota_{-}: X_{+} \amalg X_{-} \rightarrow X$ is the normalization map. Let $U=X_{\text {reg }}=\left(X_{+} \backslash D\right) \amalg\left(X_{-} \backslash D\right)$. We shall use the following important further properties of $X$.

Lemma 6.1. The scheme $X$ satisfies the following properties.

(1) It is Cohen-Macaulay.

(2) It is separably weakly normal.

(3) It is weakly normal.

(4) The map $D \rightarrow X_{+} \times_{X} X_{-}$is an isomorphism.

Proof. Because $X_{+}$and $X_{-}$are smooth of dimension $d$, they are Cohen-Macaulay. Since $D$ is an effective Cartier divisor on a smooth scheme of dimension $d$, it is Cohen-Macaulay of dimension $d-1$. The statement (1) now follows from [2, Lemma 1.2, Lemma 1.5, (1.5.3)]. Second statement follows because $D \subset X$ is the smallest conducting closed subscheme for the normalization $\pi: X^{N} \rightarrow X$ which is reduced. Furthermore, the map $D \times_{X} X^{N} \simeq D \amalg D \stackrel{\pi}{\rightarrow} D$ is just the collapse map and hence generically separable. The statement (3) follows from the previous two and (4) follows from Lemma 2.2.

6.2. The main result. We shall use the set-up of $\S 6.1$ throughout this section. We assume from now on that $d=\operatorname{dim}(X) \geq 3$. Let $C \hookrightarrow X$ be a reduced Cartier curve (see $\S 2.1$ ) such that each irreducible component of $C \backslash D$ is smooth and $C \backslash D$ has only double point singularities.

Lemma 6.2. There exists a locally closed embedding $X \hookrightarrow \mathbb{P}_{k}^{n}$ such that for a general set of distinct hypersurfaces $H_{1}, \cdots, H_{d-2} \subset \mathbb{P}_{k}^{n}$ of large degree containing $C$, the intersection $L=H_{1} \cap \cdots \cap H_{d-2}$ satisfies the following.

(1) $X \cap L$ is reduced.

(2) $D \cap L$ is reduced.

(3) $X_{ \pm} \cap L$ is an integral normal surface whose singular locus is contained in $C_{\text {sing }} \cap D$.

(4) The inclusion $C \subset(X \cap L)$ is a local complete intersection along $D$. 
Proof. Since $X$ is reduced of dimension $d \geq 3$, and since $C \subset X$ is a local complete intersection along $D=X_{\text {sing }}$, it follows from [38, Lemmas 1.3, 1.4] (see also [9, Sublemma 1]) that for all $m \gg 1$, there is an open dense subset $\mathcal{U}_{n}(C, m)$ of the scheme $\mathcal{H}_{n}(C, m)$ of hypersurfaces in $\mathbb{P}_{k}^{n}$ of degree $m$ containing $C$ such that the following hold.

a) For general distinct $H_{1}, \cdots, H_{d-2} \in \mathcal{U}_{n}(C, m)$, the scheme-theoretic intersection $L=H_{1} \cap \cdots \cap H_{d-2}$ has the property that $X \cap L$ is reduced away from $C$.

b) $D \cap L$ is reduced away from $C$.

c) $X_{ \pm} \cap L$ is integral away from $C$.

d) $C \subset(X \cap L)$ is a local complete intersection along $D$.

Note that as $X_{ \pm}$is smooth and $X_{ \pm} \cap L$ is a complete intersection in $X_{ \pm}$, it follows that $X_{ \pm} \cap L$ is a Cohen-Macaulay surface. In particular, it has no embedded component. Since $C$ is nowhere dense in $X_{ \pm} \cap L$, it follows from (c) that $X_{ \pm} \cap L$ must be irreducible. Since $C$ does not contain the generic point of $X_{ \pm} \cap L$, it follows from (b) and Lemma 6.3 that $X_{ \pm} \cap L$ is integral.

Setting $W:=C \backslash\left(C_{\text {sing }} \cap D\right)$ and following [1, $\left.\S 5\right]$, let $W\left(\Omega_{C}^{1}, e\right)$ denote the locally closed subset of points in $W$ where the embedding dimension of $W$ is $e$. It follows from our assumption on the singularities of $C \backslash D$ that $\max _{e}\left\{\operatorname{dim}\left(W\left(\Omega_{C}^{1}, e\right)\right)+e\right\} \leq 2$. We conclude from [1, Theorem 7] that for all $m \gg 1$, there is an open dense subset $\mathcal{W}_{n}(W, m)$ of the scheme $\mathcal{H}_{n}(W, m)$ of hypersurfaces of $\mathbb{P}_{k}^{n}$ of degree $m$ containing $W$ such that for general distinct $H_{1}, \cdots, H_{d-2} \in \mathcal{W}_{n}(W, m)$, the scheme-theoretic intersection $L=H_{1} \cap \cdots \cap H_{d-2}$ has the following properties.

a') $X \cap L$ is a complete intersection in $X$ of dimension two.

b') $(X \cap L) \backslash D$ is smooth.

c') $X_{ \pm} \cap L$ is smooth away from $C_{\text {sing }} \cap D$.

It follows from (c') that $X_{ \pm} \cap L$ is a Cohen-Macaulay surface whose singular locus is contained in $C_{\text {sing }} \cap D$. It follows from Serre's normality condition that $X_{ \pm} \cap L$ is normal. Since $C$ is the closure of $W$ in $X$, we must have $\mathcal{H}_{n}(W, m)=\mathcal{H}_{n}(C, m)$ and in particular, $D \subset X \cap L$. Combining (a) - (d) and (a') - (c') together, we conclude that there is a closed immersion $Y \subset X$ with the following properties.

(1) $\operatorname{dim}(Y)=2$.

(2) The inclusions $Y \subset X,\left(X_{ \pm} \cap Y\right) \subset X_{ \pm}$and $(D \cap Y) \subset D$ are all complete intersections.

(3) $Y$ is reduced away from $C \cap D$.

(4) $(D \cap Y) \subset Y$ is a Cartier divisor which is reduced away from $C$.

(5) $X_{ \pm} \cap Y$ is an integral normal surface which is smooth away from $C_{\text {sing }} \cap D$.

(6) $C \subset Y$ is a local complete intersection along $D$.

(7) $Y \backslash D$ is smooth.

If $Y \subset X$ is as above, then (6) says that the local rings of $Y$ at $C \cap D$ contain regular elements. In particular, $C \cap D$ can contain no embedded point of $Y$. We conclude from (3) and Lemma 6.3 that a surface $Y$ as above must be reduced. Since $D \subset X_{ \pm}$is a Cartier divisor, it is Cohen-Macaulay and hence (2) shows that $D \cap Y$ is a complete intersection Cohen-Macaulay curve inside $D$. Since $C \cap D$ can contain no generic point of $D \cap Y$, it follows form (4) and Lemma 6.3 that $D \cap Y$ is a reduced curve. This finishes the proof of the lemma.

The following is a straightforward application of the prime avoidance theorem in commutative algebra and its proof is left to the reader.

Lemma 6.3. Let $R$ be commutative Noetherian ring such that $R_{\mathfrak{p}}$ is reduced for every associated prime ideal $\mathfrak{p}$ of $R$. Then $R$ must be reduced. 
Lemma 6.4. Let $X=X_{+} \amalg_{D} X_{-}$be as in (6.1) and let $Y=X \cap L$ be the complete intersection surface as obtained in Lemma 6.2. Assume that $X$ is projective. Then, $A^{2}(Y)$ is a semi-abelian variety and the Roitman torsion theorem holds for $Y$. That is, the Abel-Jacobi map $\rho_{Y}: \mathrm{CH}_{0}^{L W}(Y)_{\operatorname{deg} 0} \rightarrow A^{2}(Y)$ is an isomorphism on the torsion subgroups.

Proof. We let $Y_{ \pm}=X_{ \pm} \cap Y$ and $E=D \cap Y$. Let $\iota_{ \pm}^{\prime}: Y_{ \pm} \hookrightarrow Y$ be the inclusion maps. It follows from the construction of $Y \subset X$ and an easy variant of [7, Lemma 2.2] that the canonical map $Y_{+} \amalg_{E} Y_{-} \rightarrow Y$ is an isomorphism. It also follows from Lemma 6.2 that $\iota_{+}^{\prime} \amalg \iota_{-}^{\prime}: Y_{+} \amalg Y_{-} \rightarrow Y$ is the normalization map. Since $Y$ is the join of normal surfaces $Y_{+}$and $Y_{-}$along the common closed subscheme $E$, it follows that the non-normal locus of $Y$ is the support of $E$. Let us denote the map $\iota_{+}^{\prime} \amalg \iota_{-}^{\prime}$ by $\pi^{\prime}$.

Let $\mathcal{I}_{ \pm} \subset \mathcal{O}_{Y_{ \pm}}$denote the defining ideal sheaf for the inclusion $E \subset Y_{ \pm}$. It is then immediate from (6.1) that $\pi^{\prime *}\left(\mathcal{I}_{E}\right)=\mathcal{I}_{+} \times \mathcal{I}_{-}$which is actually in $\mathcal{O}_{Y}$ under the inclusion $\pi^{\prime *}: \mathcal{O}_{Y} \hookrightarrow \pi_{*}^{\prime}\left(\mathcal{O}_{Y_{+}} \amalg Y_{-}\right)$. In other words, $E \hookrightarrow Y$ is a conducting subscheme for $\pi^{\prime}$. Since $\pi^{\prime *}(E)=E \amalg E$ is reduced by Lemma 6.2 and since the support of $E$ is the non-normal locus of $Y$, we see that $\pi^{\prime}$ is the normalization map for which $E$ is the smallest conducting subscheme and is reduced. Since $\pi^{\prime}: E \amalg E \rightarrow E$ is just the collapse map, it is clearly generically separable. We conclude (see Definition 4.5) that $Y$ is a reduced projective surface which is separably weakly normal. The lemma now follows from Theorem 4.9.

Theorem 6.5. Let $X_{+}$and $X_{-}$be two smooth projective schemes of dimension $d \geq 1$ over $k$ and let $X=X_{+} \amalg_{D} X_{-}$be as in (6.1). Then, the Albanese variety $A^{d}(X)$ is a semiabelian variety and the Abel-Jacobi map $\rho_{X}: \mathrm{CH}_{0}^{L W}(X) \operatorname{deg} 0 \rightarrow A^{d}(X)$ is isomorphism on the torsion subgroups.

Proof. It follows from Lemma 6.1 that $X$ is separably weakly normal and weakly normal. Hence, in characteristic zero, a general reduced Cartier curve on $X$ containing a 0cycle is weakly normal by [15, Corollary 2.5]. We can thus repeat the argument of the proof of Theorem 4.9, which shows that $\rho_{X}$ is surjective and reduces the remaining proof to showing only that the restriction of $\rho_{X}$ to the $p$-primary torsion subgroup of $\mathrm{CH}_{0}^{L W}(X)_{\operatorname{deg} 0}$ is injective when $p \geq 2$.

When $d=1$, the scheme $X$ is a weakly normal curve, and it is well known in this case that $\operatorname{Pic}^{0}(X) \simeq A^{1}(X) \simeq J^{1}(X)$. The case $d=2$ is Lemma 6.4. So we assume $d \geq 3$.

Let $\alpha \in \mathcal{Z}_{0}(X)$ be such that $n \alpha=0$ in $\mathrm{CH}_{0}^{L W}(X)$ for some integer $n=p^{m}$. Equivalently, $n \alpha \in \mathcal{R}_{0}^{L W}(X)$. Let us assume further that $\rho_{X}^{\text {semi }}(\alpha)=0$.

We can now use [9, Lemma 2.1] to find a reduced Cartier curve $C$ on $X$ and a function $f \in \mathcal{O}_{C, S}^{\times}$such that $n \alpha=\operatorname{div}(f)$, where $S=C \cap X_{\text {sing }}=C \cap D$. Since part of $n \alpha$ supported on any connected component of $C$ is also of the form $n \alpha^{\prime}=\operatorname{div}_{C^{\prime}}\left(f^{\prime}\right)$ for some Cartier curve $C^{\prime}$ and some $f^{\prime}$, we can assume that $C$ is connected. Let $\left\{C_{1}, \cdots, C_{r}\right\}$ denote the set of irreducible components of $C$.

We set $U=X \backslash D=\left(X_{+} \backslash D\right) \amalg\left(X_{-} \backslash D\right)$. Let $\phi: X^{\prime} \rightarrow X$ be a successive blow-up at smooth points such that the following hold.

(1) The strict transform $D_{i}$ of each $C_{i}$ is smooth along $\phi^{-1}(U)$.

(2) $D_{i} \cap D_{j} \cap \phi^{-1}(U)=\emptyset$ for $i \neq j$.

(3) Each $D_{i}$ intersects the exceptional divisor $E$ (which is reduced) transversely at smooth points.

It is clear that there exists a finite set of blown-up closed points $T=\left\{x_{1}, \cdots, x_{n}\right\} \subset U$ such that $\phi: \phi^{-1}(X \backslash T) \rightarrow X \backslash T$ is an isomorphism. In particular, $\phi: X_{\text {sing }}^{\prime}=\phi^{-1}(D) \rightarrow$ $D$ is an isomorphism. If we identify $\phi^{-1}(D)$ with $D$ and let $X_{ \pm}^{\prime}=\mathrm{Bl}_{T \cap X_{ \pm}}\left(X_{ \pm}\right)$, it becomes clear that $X^{\prime}=X_{+}^{\prime} \amalg_{D} X_{-}^{\prime}$. We set $U^{\prime}=X^{\prime} \backslash D=\phi^{-1}(U)$. Let $C^{\prime}$ denote the strict transform of $C$ with components $\left\{C_{1}^{\prime}, \cdots, C_{r}^{\prime}\right\}$. 
Since $\phi$ is an isomorphism over an open neighborhood of $D$, it follows that $\phi^{-1}(S) \simeq S$ and the map $C^{\prime} \rightarrow C$ is an isomorphism along $D$. In particular, we have $f \in \mathcal{O}_{C^{\prime}, S}^{\times}$ and $\phi_{*}\left(\operatorname{div}_{C^{\prime}}(f)\right)=\operatorname{div}_{C}(f)=n \alpha$. Since $\operatorname{Supp}(\alpha) \subset C^{\prime}$, we can find $\alpha^{\prime} \in \mathcal{Z}_{0}\left(X^{\prime}\right)$ supported on $C^{\prime}$ such that $\phi_{*}\left(\alpha^{\prime}\right)=\alpha$. This implies that $\phi_{*}\left(n \alpha^{\prime}-\operatorname{div}_{C^{\prime}}(f)\right)=0$. Setting $\beta=n \alpha^{\prime}-\operatorname{div}_{C^{\prime}}(f)$, it follows that $\beta$ must be a 0 -cycle on the exceptional divisor such that $\phi_{*}(\beta)=0$.

We can now write $\beta=\sum_{i=0}^{n} \beta_{i}$, where $\beta_{i}$ is a 0 -cycle on $X^{\prime}$ supported on $\phi^{-1}\left(x_{i}\right)$ for $1 \leq i \leq n$ and $\beta_{0}$ is supported on the complement $E$. We must then have $\phi_{*}\left(\beta_{i}\right)=0$ for all $0 \leq i \leq n$. Since $\phi$ is an isomorphism away from $T=\left\{x_{1}, \cdots, x_{n}\right\}$, we must have $\beta_{0}=0$. We can therefore assume that $\beta$ is a 0 -cycle on $E$.

Next, we note that each $\phi^{-1}\left(\left\{x_{i}\right\}\right)$ is a $(d-1)$-dimensional projective variety whose irreducible components are point blow-ups of $\mathbb{P}_{k}^{d-1}$, intersecting transversely in $X_{\text {reg }}^{\prime}$. Moreover, we have $\phi_{*}\left(\beta_{i}\right)=0$ for the push-forward map $\phi_{*}: \mathcal{Z}_{0}\left(\pi^{-1}\left(\left\{x_{i}\right\}\right)\right) \rightarrow \mathbb{Z}$, induced by $\phi: \phi^{-1}\left(\left\{x_{i}\right\}\right) \rightarrow \operatorname{Spec}\left(k\left(x_{i}\right)\right)=k$. But this means that $\operatorname{deg}(\beta)=\sum_{i=0}^{n} \operatorname{deg}\left(\beta_{i}\right)=0$. In particular, there are finitely many smooth projective rational curves $L_{j} \subset E$ and rational functions $g_{j} \in k\left(L_{j}\right)$ such that $\beta=\sum_{j=1}^{s}\left(g_{j}\right)_{L_{j}}$.

Using the argument of [11, Lemma 5.2], we can further choose $L_{j}$ 's so that $C^{\prime \prime}:=$ $C^{\prime} \cup\left(\cup_{j} L_{j}\right)$ is a connected reduced curve with following properties.

(1) Each component of $C^{\prime \prime}$ is smooth along $U^{\prime}$.

(2) $C^{\prime \prime} \cap U^{\prime}$ has only ordinary double point singularities.

In particular, the embedding dimension of $C^{\prime \prime}$ at each of its singular points lying over $U$ is two. Furthermore, $C^{\prime \prime} \cap D=\left(C^{\prime \prime} \backslash\left(\cup_{j} L_{j}\right)\right) \cap D=C^{\prime} \cap D$. This implies that $C^{\prime \prime}$ is a Cartier curve on $X^{\prime}$.

We now fix a closed embedding $X^{\prime}=X_{+}^{\prime} \amalg_{D} X_{-}^{\prime} \hookrightarrow \mathbb{P}_{k}^{N}$ and choose a complete intersection surface $j^{\prime}: Y^{\prime} \subset X^{\prime}$ as in Lemma 6.2. Let $\pi^{\prime}: X_{+}^{\prime} \amalg X_{-}^{\prime} \rightarrow X^{\prime}$ denote the normalization map with $\pi^{\prime-1}\left(Y^{\prime}\right)=Y_{+}^{\prime} \amalg Y_{-}^{\prime}$. Note that $E^{\prime}=Y^{\prime} \cap D$ is a reduced curve and $Y_{\text {sing }}^{\prime}=E^{\prime}$. Since $C^{\prime \prime} \cap E^{\prime}=C^{\prime} \cap E^{\prime}$ and since $C^{\prime \prime}$ is Cartier on $Y^{\prime}$, it follows that $C^{\prime}$ and $L_{j}$ 's are also Cartier curves on $Y^{\prime}$. Furthermore, $\alpha^{\prime}$ is an element of $\mathcal{Z}_{0}\left(Y^{\prime}, E^{\prime}\right)$ such that $n \alpha^{\prime}=\operatorname{div}_{C^{\prime}}(f)+\sum_{j} \operatorname{div}_{L_{j}}\left(g_{j}\right)$. In particular, $\alpha^{\prime} \in \mathrm{CH}_{0}^{L W}\left(Y^{\prime}\right)$ and $n \alpha^{\prime}=0$ in $\mathrm{CH}_{0}^{L W}\left(Y^{\prime}\right)$. Note that this also implies that $\alpha^{\prime} \in \mathrm{CH}_{0}^{L W}\left(Y^{\prime}\right)_{\operatorname{deg} 0}$.

It follows from [42, Theorem 14] that there exists a commutative diagram

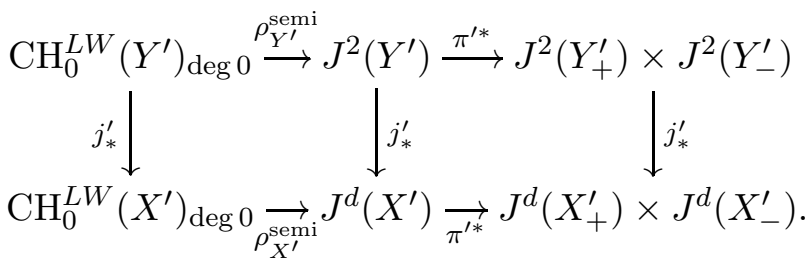

Since $\phi: X^{\prime} \rightarrow X$ is a blow-up at smooth closed points and since the Albanese variety of a smooth projective scheme is a birational invariant, it follows from the construction of $J^{d}(X)$ in $\S 2.4$ and [18, Lemma 2.5] that the canonical push-forward map $\phi_{*}: \mathcal{Z}_{0}\left(X^{\prime}\right) \rightarrow$ $\mathcal{Z}_{0}(X)$ gives rise to a commutative diagram 


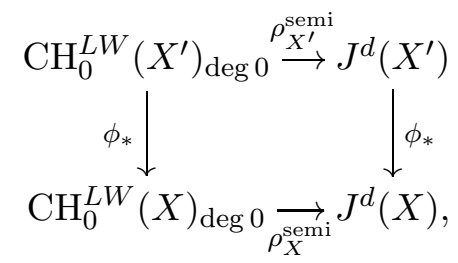

in which the two vertical arrows are isomorphisms. It follows that $\alpha^{\prime} \in \mathrm{CH}_{0}^{L W}\left(Y^{\prime}\right) \operatorname{deg} 0$ is a 0 -cycle such that $\rho_{X^{\prime}}^{\text {semi }} \circ j_{*}^{\prime}\left(\alpha^{\prime}\right)=0$ in $J^{d}\left(X^{\prime}\right)$. Using (6.2), we conclude that

$$
j_{*}^{\prime} \circ \pi^{\prime *} \circ \rho_{Y^{\prime}}^{\mathrm{semi}}\left(\alpha^{\prime}\right)=\pi^{\prime *} \circ \rho_{X^{\prime}}^{\mathrm{semi}} \circ j_{*}^{\prime}\left(\alpha^{\prime}\right)=0 .
$$

Since $Y_{+}^{\prime} \amalg Y_{-}^{\prime} \rightarrow Y^{\prime}$ is the normalization map (see Lemma 6.4), we know that $J^{2}\left(Y_{+}^{\prime}\right) \times J^{2}\left(Y_{-}^{\prime}\right)$ is the universal abelian variety quotient of $J^{2}\left(Y^{\prime}\right)$. In particular, the map $J^{2}\left(Y^{\prime}\right)\{p\} \rightarrow J^{2}\left(Y_{+}^{\prime}\right)\{p\} \times J^{2}\left(Y_{-}^{\prime}\right)\{p\}$ is an isomorphism. On the other hand, $Y_{ \pm}^{\prime} \subset X_{ \pm}^{\prime}$ are the iterated hypersurface sections of normal projective schemes and hence it follows from [35. Chap. 8, $\S 2$, Theorem 5] that the right vertical arrow in (6.2) is an isomorphism of abelian varieties. Note here that $X^{\prime}$ or $Y^{\prime}$ need not be smooth for this isomorphism. It follows therefore from (6.4) that $\rho_{Y^{\prime}}^{\mathrm{semi}}\left(\alpha^{\prime}\right)=0$. Lemma 6.4 now implies that $\alpha^{\prime}=0$ and we finally get $\alpha=\phi_{*}\left(\alpha^{\prime}\right)=0$.

6.3. Applications of Theorem 6.5. We now obtain some applications of Theorem 6.5. Our first result is the following comparison theorem.

Theorem 6.6. Let $X_{+}$and $X_{-}$be two smooth projective schemes of dimension $d \geq 1$ over $k$ and let $X=X_{+} \amalg_{D} X_{-}$be as in (6.1). Then, the canonical map $\mathrm{CH}_{0}^{L W}(X) \rightarrow$ $\mathrm{CH}_{0}(X)$ is an isomorphism.

Proof. Let $L$ denote the kernel of the surjection $\mathrm{CH}_{0}^{L W}(X) \rightarrow \mathrm{CH}_{0}(X)$. Using the factorization $\mathrm{CH}_{0}^{L W}(X) \rightarrow \mathrm{CH}_{0}(X) \rightarrow K_{0}(X)$ (see [7, Lemma 3.13] and [38, Corollary 2.7]), we know that $L$ is a torsion subgroup of $\mathrm{CH}_{0}^{L W}(X)_{\operatorname{deg} 0}$ of bounded exponent. On the other hand, we also have a factorization $\mathrm{CH}_{0}^{L W}(X)_{\operatorname{deg} 0} \rightarrow \mathrm{CH}_{0}(X)_{\operatorname{deg} 0} \rightarrow J^{d}(X)$ by (2.4). Since $J^{d}(X)$ is the universal semi-abelian variety quotient of $A^{d}(X)$, we can use Theorem 6.5 to replace $J^{d}(X)$ by $A^{d}(X)$. We therefore have a factorization $\mathrm{CH}_{0}^{L W}(X)_{\operatorname{deg} 0} \rightarrow \mathrm{CH}_{0}(X)_{\operatorname{deg} 0} \rightarrow A^{d}(X)$ of $\rho_{X}^{\text {semi }}$. Another application of Theorem 6.5 now shows that $L$ is torsion-free. Hence, it must be zero.

As second application of Theorem 6.5. we now prove the Roitman torsion theorem for the Chow group of 0-cycles with modulus when the underlying divisor is reduced.

Theorem 6.7. Let $X$ be a smooth projective scheme of dimension $d \geq 1$ over $k$ and let $D \subset X$ be an effective Cartier divisor. Assume that $D$ is reduced. Then, the Albanese variety with modulus $A^{d}(X \mid D)$ is a semi-abelian variety and the Abel-Jacobi map $\rho_{X \mid D}$ : $\mathrm{CH}_{0}(X \mid D)_{\operatorname{deg} 0} \rightarrow A^{d}(X \mid D)$ is isomorphism on the torsion subgroups.

Proof. By Theorem 6.6 and [7, (11.2)], there is a commutative diagram of split exact sequences:

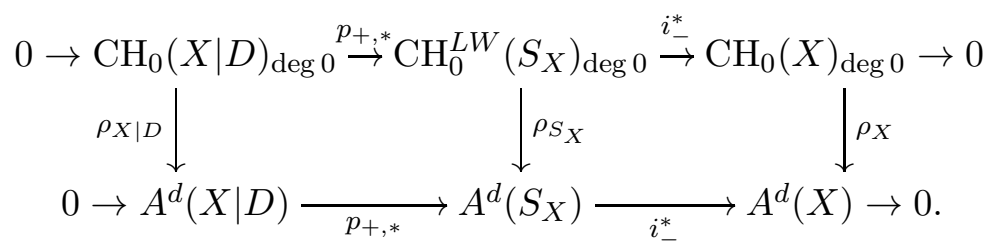

We note here that the constructions of [7, $\S 11]$ are based on the assumption that Theorem 6.6 holds. Since $X$ is smooth over $k$, we know that $A^{d}(X)$ is an abelian variety. 
Moreover, the right vertical arrow is isomorphism on the torsion subgroups by Roitman [54] and Milne [48]. We conclude that the theorem is equivalent to showing that $A^{d}\left(S_{X}\right)$ is a semi-abelian variety and $\rho_{S_{X}}$ is isomorphism on the torsion subgroups. But this follows from Theorem 6.5.

Corollary 6.8. Let $X$ be a smooth projective scheme of dimension $d \geq 1$ over $\overline{\mathbb{F}}_{p}$ and let $D \subset X$ be a reduced effective Cartier divisor. Then, $\mathrm{CH}_{0}(X \mid D)_{\operatorname{deg} 0}$ is finite-dimensional. That is, the Abel-Jacobi map $\rho_{X}: \mathrm{CH}_{0}(X \mid D)_{\operatorname{deg} 0} \rightarrow A^{d}(X \mid D)$ is an isomorphism.

Proof. In view of Theorem 6.7, we only need to show that $\mathrm{CH}_{0}\left(S_{X}\right)$ is a torsion abelian group. But this is already shown in the proof of Corollary 4.10 .

Acknowledgments. The author would like to thank the referee for very carefully reading the paper and providing many suggestions to improve its presentation.

\section{REFERENCES}

[1] A. Altman, S. Kleiman, Bertini theorems for hypersurface sections containing a subscheme, Comm. Alg., 7, (1979), no. 8, 775-790.

[2] H. Ananthnarayan, L. Avramov, F. Moore, Connected sums of Gorenstein local rings, J. Reine Angew. Math., 667, (2012), 149-176.

[3] L. Barbieri-Viale, C. Pedrini, C. Weibel, Roitman's Theorem for singular Varieties, Duke Math. J., 84, (1996), 155-190.

[4] L. Barbieri-Viale, V. Srinivas, Albanese and Picard 1-motives, Mémoires de la SMF, 87, (2001).

[5] H. Bass, Algebraic K-theory, W. A. Benjamin, Inc., New York-Amsterdam, (1968).

[6] H. Bass, M. Murthy, Grothendieck groups and Picard groups of abelian group rings, Ann. of Math., 86, (1967), 16-73.

[7] F. Binda, A. Krishna, Zero cycles with modulus and zero cycles on singular varieties, Comp. Math., 154, (2018), 120-187.

[8] F. Binda, S. Saito, Relative cycles with moduli and regulator maps, J. Math. Inst. Jussieu, (to appear), (2017), arXiv:1412.0315.

[9] J. Biswas, V. Srinivas, Roitman's theorem for singular projective varieties, Comp. Math., 119, (1999), 213-237.

[10] S. Bloch, Torsion algebraic cycles and a theorem of Roitman, Comp. Math., 39, (1979), $107-127$.

[11] S. Bloch, Lectures on algebraic cycles, Duke University Math. Series, 4, (1980).

[12] S. Bloch, Algebraic Cycles and Higher K-theory, Adv. Math., 61, (1986), 267-304.

[13] S. Bloch, A. Ogus, Gersten's conjecture and the homology of schemes, Ann. scient. Éc. Norm. Sup., 7, No. 2, (1974), 181-201.

[14] K. Brown, S. Gersten, Algebraic K-theory as generalized sheaf cohomology, LNM series, 341, Springer-Verlag, Berlin, (1973), 266-292.

[15] C. Cumino, S. Greco, M. Manaresi, Bertini theorems for weak normality, Comp. Math., 48, (1983), 351-362.

[16] C. Cumino, S. Greco, M. Manaresi, Hyperplane sections of weakly normal varieties in positive characteristic, Proc. AMS, 106, (1989), 37-42.

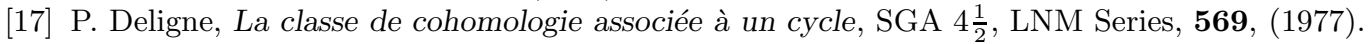

[18] H. Esnault, V. Srinivas, E. Viehweg, The Universal regular quotient of Chow group of points on projective varieties, Invent. Math., 135, (1999), 595-664.

[19] K. Fujiwara, A proof of the absolute purity conjecture, Adv. Stud. in Pure Math., Math. Soc. Japan, 36, (2002), 144-173.

[20] W. Fulton, Intersection theory, Second Edition, Ergebnisse der Mathematik und ihrer Grenzgebiete 3, Folge. A Series of Modern Surveys in Mathematics, 2, Springer-Verlag, Berlin, (1998).

[21] S. Geller, C. Weibel, $K_{1}(A, B, I)$, J. Reine Angew. Math., 342, (1983), 12-34.

[22] H. Gillet, Riemann-Roch Theorems for higher algebraic K-theory, Adv. Math., 40, (1981), $203-289$.

[23] H. Gillet, Gersten's conjecture for the $K$-theory with torsion coefficients of a discrete valuations ring, J. Alg., 103, (1986), 377-380.

[24] D. Grayson, Universal exactness in algebraic K-theory, J. Pure Appl. Algebra, 36, (1985), $139-141$.

[25] S. Greco, C. Traverso, On seminormal schemes, Comp. Math., 40, (1980), 325-365.

[26] R. Hoobler, The Merkurjev-Suslin theorem for any semi-local ring, J. Pure Appl. Algebra, 207, (2006), no. 3, 537-552.

[27] C. Huneke, I. Swanson, Integral closure of Ideals, Rings, and Modules, London Math. Soc., Lecture Note Series, 336, Cambridge University Press. 
[28] R. Iwasa, W. Kai, Chern classes with modulus, arXiv:1611.07882, (2016).

[29] M. Kerz, S. Saito, Chow group of 0-cycles with modulus and higher dimensional class field theory, Duke Math. J., 165, (2016), no. 15, 2811-2897.

[30] A. Krishna, On 0-cycles with modulus, Algebra \& Number Theory, 9, (2015), no. 10, 2397-2415.

[31] A. Krishna, Zero-cycles on affine varieties, arXiv:1511.04221 v1, (2015).

[32] A. Krishna, M. Levine, Additive higher Chow groups of schemes, J. Reine Angew. Math., 619, (2008), 75-140.

[33] A. Krishna, J. Park, A module structure and a vanishing theorem for cycles with modulus, Math. Res. Lett., 24, (2017), 1147-1176.

[34] A. Krishna, V. Srinivas, Zero-Cycles and K-theory on normal surfaces, Ann. of Math., 156, (2002), $155-195$.

[35] S. Lang, Abelian Varieties, Interscience Tracts in Pure and Applied Math., 7, (1959).

[36] M. Levine, A geometric theory of the Chow ring of a singular variety, Unpublished manuscript.

[37] M. Levine, Bloch's formula for singular surfaces, Topology, 24, (1985), 165-174.

[38] M. Levine, Zero-cycles and $K$-theory on singular varieties, Proc. Symp. Pure Math., 46, Amer. Math. Soc., Providence, (1987), 451-462.

[39] M. Levine, Mixed motives, Mathematical surveys and monographs, 57, Amer. Math. Soc., (1998).

[40] M. Levine, The indecomposable $K_{3}$ of fields, Ann. scient. Éc. Norm. Sup., 22, (1989), 255-344.

[41] M. Levine, C. Weibel, Zero cycles and complete intersections on singular varieties, J. Reine Angew. Math., 359, (1985), 106-120.

[42] V. Mallick, Roitman's theorem for singular projective varieties in arbitrary characteristic, J. KTheory 3, (2009), 501-531.

[43] M. Manaresi, Some properties of weakly normal varieties, Nagoya Math. J., 77, (1980), 61-74.

[44] H. Matsumura, Commutative ring theory, Cambridge studies in advanced mathematics, 8, (1997), Cambridge University Press, Cambridge.

[45] J. P. May, Simplicial objects in algebraic topology, Van Nostrand Math. Stud., 11, Van Nostrand, Princeton, (1967).

[46] A. Merkurjev, A. Suslin, The group $K_{3}$ of a field, Math. USSR Izvestiya, 36, (1991), 541-565.

[47] J. Milne, Étale Cohomology, Princeton Math. Ser., 33, Princeton University Press, (1980).

[48] J. Milne, Zero cycles on algebraic varieties in nonzero characteristic: Roitman's theorem, Comp. Math., 47, (1982), Fasc. 3, 271-287.

[49] H. Miyazaki, Cube invariance of higher Chow groups with modulus, arXiv:1604.06155, (2017).

[50] A. Navarro, Riemann-Roch for homotopy invariant $K$-theory and Gysin morphisms, arXiv:1605.00980, (2016).

[51] J. Park, Regulators on additive higher Chow groups, Amer. J. Math., 131, (2009), no. 1, $257-276$.

[52] C. Pedrini, C. Weibel, Divisibility in the Chow group of zero-cycles on a singular surface, Asterisque 226 (1994) 371-409.

[53] D. Quillen, Higher Algebraic K-theory, LNM Series, 341, Springer-Verlag, Berlin, (1973), 85-147.

[54] A. Roitman, The torsion in the group of 0-cycles modulo rational equivalence, Ann. Math., 111, (1980), 553-569.

[55] K. Rülling, The generalized de Rham-Witt complex over a field is a complex of zero-cycles, J. Alg. Geom., 16, no. 1, (2007), 109-169.

[56] J.-P. Serre, Morphisms universels et differéntielles de troisiéme espéce, Seminaire Chevalley, (1958).

[57] A. Suslin, Torsion in $K_{2}$ of fields, $K$-Theory, 1, (1987), 5-29.

[58] C. Traverso, Seminormality and Picard group, Annali della Scuola Normale Superiore di Pisa Classe di Scienze, Série 3, 24, (1970), no. 4, 585-595.

[59] M. Vitulli, Weak normality and semi-normality, in "Commutative algebra-Noetherian and nonNoetherian perspectives", Springer, New York, (2011), 441-480.

[60] V. Voevodsky, Cohomological theory of presheaves with transfers, in "Cycles, transfers and motivic homology theories", volume 143 of Ann. of Math. Stud., Princeton Univ. Press, Princeton, NJ, 143, (2000), 87-737.

[61] A. Weil, Sur les critéres d'équivalence en géométrique algébrique, Math. Annalen, 128, (1954), 209-215.

[62] H. Yanagihara, Some results on weakly normal ring extensions, J. Math. Soc. Japan, 35, (1983), 649-661.

School of Mathematics, Tata Institute of Fundamental Research, 1 Homi Bhabha Road, Colaba, Mumbai, India

E-mail address: amal@math.tifr.res.in 